\title{
Interventions for renal vasculitis in adults. A systematic review
}

\author{
Giles D Walters ${ }^{* 1}$, Narelle S Willis² and Jonathan C Craig ${ }^{3}$
}

\begin{abstract}
Background: Renal vasculitis presents as rapidly progressive glomerulonephritis and comprises of a group of conditions characterised by acute kidney failure, haematuria and proteinuria. Treatment of these conditions involves the use of steroid and non-steroid agents with or without adjunctive plasma exchange. Although immunosuppression has been successful, many questions remain unanswered in terms of dose and duration of therapy, the use of plasma exchange and the role of new therapies. This systematic review was conducted to determine the benefits and harms of any intervention for the treatment of renal vasculitis in adults.

Methods: We searched the Cochrane Central Register of Controlled Trials, the Cochrane Renal Group Specialised Register, MEDLINE and EMBASE to June 2009. Randomised controlled trials investigating any intervention for the treatment of adults were included. Two authors independently assessed study quality and extracted data. Statistical analyses were performed using a random effects model and results expressed as risk ratio with 95\% confidence intervals for dichotomous outcomes or mean difference for continuous outcomes.

Results: Twenty two studies (1674 patients) were included. Plasma exchange as adjunctive therapy significantly reduces the risk of end-stage kidney disease at 12 months (five studies: RR $0.47, \mathrm{Cl} 0.30$ to 0.75 ). Four studies compared the use of pulse and continuous administration of cyclophosphamide. Remission rates were equivalent but pulse treatment causes an increased risk of relapse (4 studies: RR 1.79, Cl 1.11 to 2.87) compared with continuous cyclophosphamide. Azathioprine has equivalent efficacy as a maintenance agent to cyclophosphamide with fewer episodes of leukopenia. Mycophenolate mofetil may be equivalent to cyclophosphamide as an induction agent but resulted in a higher relapse rate when tested against Azathioprine in remission maintenance. Rituximab is an effective remission induction agent. Methotrexate or Leflunomide are potential choices in remission maintenance therapy. Oral co-trimoxazole did not reduce relapses significantly in Wegener's granulomatosis.
\end{abstract}

Conclusions: Plasma exchange is effective in patients with severe ARF secondary to vasculitis. Pulse cyclophosphamide results in an increased risk of relapse when compared to continuous oral use but a reduced total dose. Whilst cyclophosphamide is standard induction treatment, rituximab and mycophenolate mofetil are also effective. Azathioprine, methotrexate and leflunomide are effective as maintenance therapy. Further studies are required to more clearly delineate the appropriate place of newer agents within an evidence-based therapeutic strategy.

\section{Background}

Renal vasculitis presents as rapidly progressive glomerulonephritis (RPGN) which comprises a group of conditions characterised by acute kidney failure (AKF), haematuria and proteinuria. Histological examination of the kidney reveals severe inflammation in the form of

* Correspondence: giles.walters@act.gov.au

1 Senior Staff Specialist, The Canberra Hospital, Canberra, Australia. Hon. Senior Lecturer, Australian National University Medical School, Canberra, Australia Full list of author information is available at the end of the article crescent formation, glomerular necrosis and vasculitis of small and medium sized vessels within the kidney. These conditions include the anti-neutrophil cytoplasmic antibody (ANCA) associated vasculitides (AAV), anti-glomerular basement membrane (anti-GBM) disease and idiopathic RPGN [1]. AAV are generally small vessel vasculitides and include Wegener's granulomatosis, microscopic polyarteritis and renal limited vasculitis [2,3]. Evidence increasingly points to the pathogenicity of ANCA [4]. Other conditions also cause vasculitis in the 
kidney such as Henoch Schonlein Purpura and cryoglobulinaemia resulting in immune deposits visible on electron microscopic examination of renal tissue. The treatment of Goodpasture's disease and other forms of RPGN with granular immune deposits have an entirely separate pathogenesis to the pauci-immune (no immune deposits) forms of the disease and will not be addressed in this review.

The treatment of systemic and renal vasculitis involves the use of steroids in combination with other non-steroid agents, most commonly, cyclophosphamide (CPA) [5,1] to induce remission of disease. In the presence of kidney failure, plasma exchange is often used as an adjunct to pharmacological treatment [6-8]. Once remission is achieved, treatment is scaled back to maintenance therapy with lower doses of steroids and CPA is replaced by a less potent immunosuppressive, such as azathioprine (AZA). Co-trimoxazole has been used in Wegener's granulomatosis mainly to prevent the occurrence of pneumocystis infection, upper respiratory tract infection and subsequent relapse of disease. Various guidelines are available which summarise available treatment options and some of the evidence for their use[9-11]

These treatments are well established but many questions remain unanswered [12]. Optimal agent, dose, duration, route and frequency of treatment are uncertain. CPA can be given as a daily oral dose or as intermittent oral or intravenous doses. Intravenous regimens tend to give a lower total dose and have fewer side effects, but may later give a higher rate of relapse [13]. Treatment may include intravenous methylprednisolone or plasma exchange but their place in therapy is debated $[14,15]$. Other therapies including Mycophenolate mofetil (MMF), anti-TNF therapy, leflunomide, methotrexate, anti-CD52 therapy, B cell depletion therapy and intravenous immunoglobulin have been suggested [16-18] but their place is not yet clear.

A previous version of the review has been published in the Cochrane library[19]. This did not include the recent data on the use of pulse cyclophosphamide[20], mycophenolate mofetil[21], leflunomide[22], methotrexate [23], rituximab[24,25] and etanercept[26]. The most notable of these is the data on pulse versus continuous cyclophosphamide and its contribution to the current debate on whether a reduction in total dose of cyclophosphamide will increase the rate of relapse. This question cannot currently be answered by the randomised controlled trials. This systematic review data is currently the best available evidence to contribute to that debate.

\section{Methods Objectives}

To evaluate the benefits and harms of any intervention used for the treatment of renal vasculitis in adults.

\section{Studies}

All RCTs and quasi-RCTs looking at any intervention used for the treatment of renal vasculitis in adults. QuasiRCTs were included in the protocol because we expected few RCTs to be identified. Foreign language studies were not excluded.

\section{Participants}

All adults suffering from an episode of AKF and/or proteinuria and haematuria with a kidney biopsy showing severe acute glomerulonephritis with crescents, glomerular necrosis or other histological evidence of vasculitis. AKF was as defined by the included studies.

\section{Outcome measures}

Mortality, kidney function, need for renal replacement therapy (RRT), number of patients relapsing, adverse effects of each intervention.

\section{Search methods}

Relevant studies were obtained form the following sources (see Appendix 1 for more detail):

1. The Cochrane Renal Group's specialised register and the Cochrane Central Register of Controlled Trials (CENTRAL) in The Cochrane Library, (Issue 1, 2008).

2. MEDLINE (1966 to July 2009) using the optimally sensitive strategy developed for the Cochrane Collaboration for the identification of RCTs ([27])

3. EMBASE (1980 to July 2009) using a search strategy adapted from that developed for the Cochrane Col-

laboration for the identification of RCTs ([28]

4. Reference lists of nephrology textbooks, review articles and relevant studies.

\section{Included and excluded studies}

The review was undertaken by three authors (GW, NW, JC). The titles and abstracts were screened independently by GW and NW who independently assessed abstracts. Disagreements were resolved in consultation with JC.

\section{Study quality}

The quality of studies to be included were assessed independently by GW and NW without blinding to authorship or journal of publication using the check list designed by the Cochrane Renal Group. Discrepancies were resolved in discussion with JC. The quality items to be assessed are allocation concealment, blinding, intention to treat analysis, and completeness of follow-up. Blinding was assessed for investigators, participants, outcome assessors and data analysis.

\section{Statistical assessment}

For dichotomous outcomes results were expressed as a risk ratio (RR) with 95\% confidence intervals (CI). Data 
were pooled using the random effects model but the fixed effects model were also analysed to ensure robustness of the model chosen and susceptibility to outliers. Where continuous scales of measurement were used to assess the effects of treatment, the mean difference (MD) was used, or the standardised mean difference (SMD) if necessary. Heterogeneity was analysed using $X^{2}$ test on N-1 degrees of freedom, with an $\alpha$ a of 0.05 used for statistical significance and with the $I^{2}$ test (Higgins 2003). $I^{2}$ values of $25 \%, 50 \%$ and $75 \%$ correspond to low, medium and high levels of heterogeneity.

The summary measure data were translated into number needed to treat (NNT) and number needed to harm $(\mathrm{NNH})$ for the observed overall baseline risks. Adverse effects were tabulated and assessed with descriptive techniques.

\section{Results}

\section{Description of studies}

Twenty two studies (28 references, 1674 participants) were identified as eligible for inclusion in this review. For remission induction, six studies assess the use of plasma exchange adjunctive therapy [7,29-33], four studies address the use of pulse versus continuous cyclophosphamide treatment [20,34-36] and six further studies consider other potential treatments including rituximab[24,25], mycophenolate[21], lymphocytapheresis[37], immunoadsorption [38] for remission induction or intravenous immunoglobulin for refractory disease[16]. Maintenance treatment is considered by six studies including comparisons of AZA after 3 months of remission induction with continued $\mathrm{CPA}[39], \mathrm{AZA}$ and MMF[40], AZA and Methotrexate[23], Methotrexate and Leflunomide[22], the use of co-trimoxazole[41] and etanercept[26]. No quasi RCT studies were identified. The demographics of the included patients are included in the review in the Cochrane Library. The numbers of studies identified by the search and subsequently excluded are documented in the flow diagram in Figure 1

\section{Plasma Exchange}

Six studies addressed the adjunctive use of plasma exchange with immunosuppression [29-33]. Inclusion criteria are summarised in Table 1 . The treatments tested in each of these studies differed in terms of both the standard immunosuppression and the protocol for plasma exchange (Table 2).

Plasma exchange significantly reduced the need for dialysis at three months (1 study; RR $0.4595 \%$ CI 0.24 to $0.84 ; \mathrm{P}=0.01 ; \mathrm{NNT}=5$ ) and 12 months (5 studies; RR $0.47 ; 95 \%$ CI 0.30 to $0.75 ; \mathrm{P}=0.002 ; \mathrm{NNT}=5 ; \mathrm{I}^{2}=0 \%$ ) post-treatment (Figure 2). Jayne 2007 included patients with $\mathrm{SCr}>500 \mathrm{uM}$ and showed a significant reduction in the need for dialysis at three and 12 months. For all other outcomes (death, $\mathrm{SCr}$ and side effects) there was no significant difference between the treatment groups.

\section{Pulse versus continuous cyclophosphamide}

Four studies assessed pulse versus continuous administration of CPA [20,34-36]. Systemic, rather than specifically renal, vasculitis was included. Raw data has been obtained from Adu et al and those patients with polyarteritis nodosa have been excluded from this analysis. Inclusion criteria are summarised in Table 3. The treatment regimens in these studies also differed, as summarised in Table 4.

Mortality was not significantly different between the two groups. There was evidence of statistically significant heterogeneity between the studies (Figure 3 ). There is a trend towards an increase in the number of patients requiring RRT with pulse CPA therapy, with twice as many patients requiring dialysis on this treatment compared to continuous treatment (Figure 4).

Remission rates were equivalent for the two interventions (Figure 5; 3 studies; RR 0.99, 95\% CI 0.96 to 1.03 ; P = $0.17 ; \mathrm{I}^{2}=34 \%$ ). There was an increased risk of relapse with pulse versus continuous therapy (Figure 6; 4 studies; RR $1.79,95 \%$ CI 1.11 to $2.87 ; \mathrm{P}=0.02$; $\mathrm{NNH}=5 ; \mathrm{I}^{2}=0 \%$ ).

Leukopenia was less common with pulse treatment (4 studies; RR 0.53, 95\% CI 0.36 to $0.77 ; \mathrm{P}=0.0009 ; \mathrm{NNH}=$ 5 ; $\mathrm{I}^{2}=0 \%$ ), and nausea more common (2 studies; RR 2.51, 95\% CI 1.07 to $5.89 ; \mathrm{P}=0.04 \mathrm{NNH}=7 ; \mathrm{I}^{2}=0 \%$ ). Other trends suggested less treatment failure but more serious infections with continuous therapy but these were not statistically significant. There was no significant difference in mortality and SCr.

\section{Other remission induction studies}

Inclusion and exclusion criteria, interventions and primary outcomes for these studies are detailed in tables 5 and 6.

\section{Rituximab vs cyclophosphamide alone for remission induction}

Two studies have been published in abstract form, the RITUXVAS and RAVE studies[25,24]. In RITUXVAS, 44 patients with newly diagnosed AAV with renal involvement were randomised 3:1 to receive rituximab or CPA. Four doses of Rituximab were given with 2 doses of CPA in the rituximab arm of the study. Primary end point was sustained remission at 6 months. There was no difference demonstrated for any outcome including remission and adverse events.

The RAVE study distinguished itself by being a randomised blinded study involving the use of placebo rituximab infusions and CPA and AZA tablets. One hundred and ninety seven patients with severe AAV were recruited. Only 99 of these patients were classed as hav- 


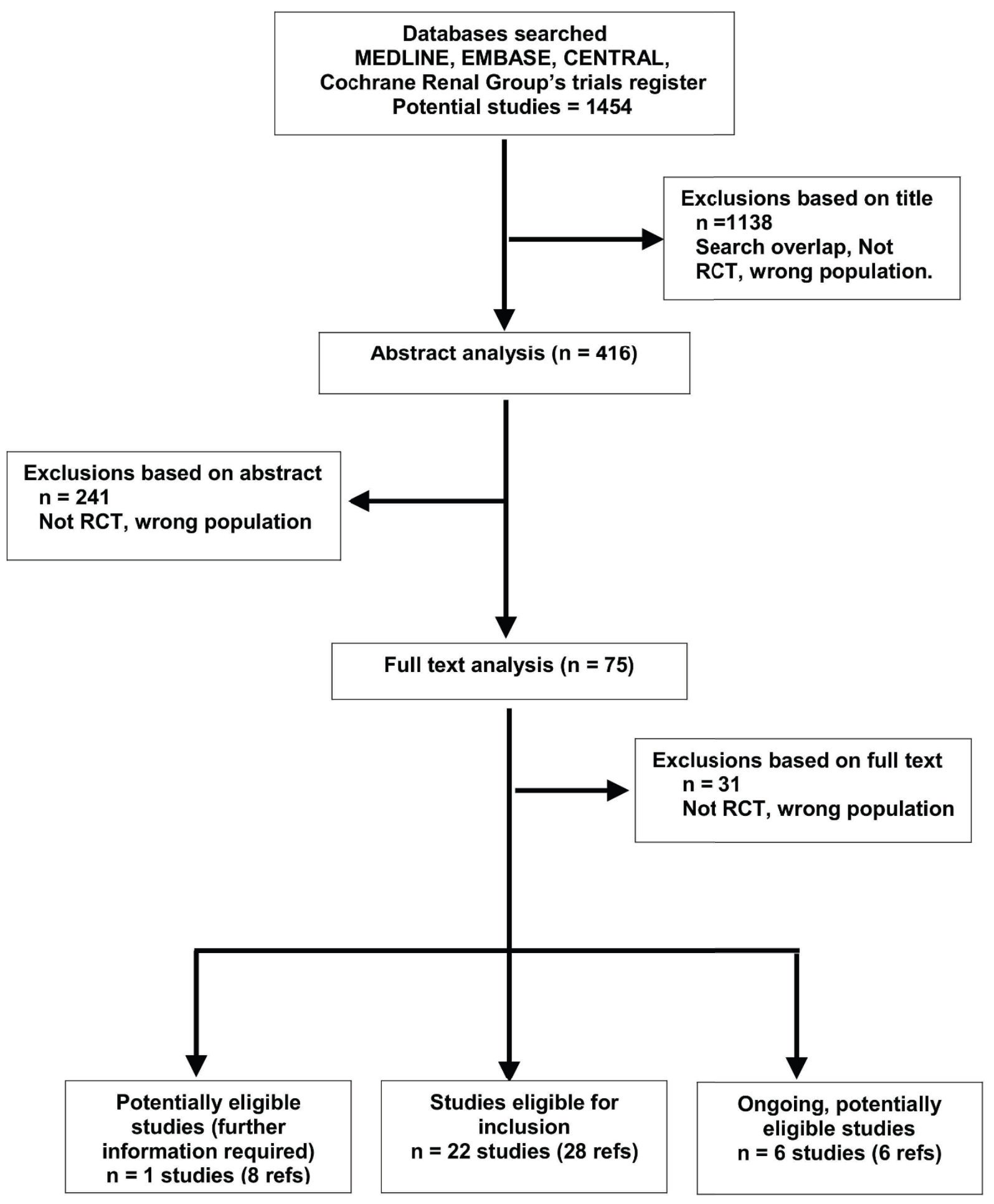


Table 1: Inclusion and exclusion criteria for plasma exchange studies

\begin{tabular}{|c|c|c|}
\hline Study Id & Inclusion criteria & Exclusion criteria \\
\hline \multirow[t]{4}{*}{ Cole 1992} & $\begin{array}{l}\text { RPGN of undefined aetiology (idiopathic or post } \\
\text { infectious disease) with specific pathologic criteria. }\end{array}$ & $\begin{array}{l}\text { Cellular crescents in }<50 \% \text { non-obsolescent glomeruli. } \\
\text { Evidence of serious infection or active ulcer disease. }\end{array}$ \\
\hline & $\begin{array}{l}\text { Adults ( } 16-75 \text { y), normal sized kidneys SCr }>170 \mu \mathrm{mol} / \mathrm{L} \\
\text { and/or increasing by } 44 \mu \text { moles/l per wk. }\end{array}$ & \\
\hline & $\begin{array}{l}\text { No evidence of systemic disease or anti-glomerular } \\
\text { basement membrane antibody-induced disease. }\end{array}$ & \\
\hline & Renal biopsy within 5 days of trial entry & \\
\hline \multirow[t]{3}{*}{ Jayne 2007} & $\begin{array}{l}\text { Biopsy-proven ANCA-associated necrotizing GN with } \\
\text { acute kidney failure }(\mathrm{SCr}>500 \mu \mathrm{mol} / \mathrm{L})\end{array}$ & Age $<18$ or $>80$ years. \\
\hline & & $\begin{array}{l}\text { Inadequate contraception; pregnancy; previous } \\
\text { malignancy; hepatitis B antigenaemia or hepatitis C } \\
\text { antibody or HIV infection; other multi-system } \\
\text { autoimmune disease; circulating anti-GBM antibody or } \\
\text { linear staining of GBM on histology; life-threatening non- } \\
\text { renal manifestations of vasculitis. }\end{array}$ \\
\hline & & $\begin{array}{l}\text { Dialysis for }>2 \text { weeks before entry; creatinine }>200 \text { uM } \\
\text { more than } 1 \text { year before entry. }>2 \text { weeks treatment with } \\
\text { CPA or AZA; }>500 \text { mg of IV methylprednisolone; plasma } \\
\text { exchange within the preceding year; }>3 \text { months } \\
\text { treatment with oral prednisolone; allergy to study } \\
\text { medications. }\end{array}$ \\
\hline Glockner 1988 & $\begin{array}{l}\text { RPGN with }>70 \% \text { crescents on renal biopsy. } \\
\mathrm{CrCl}<50 \mathrm{ml} / \mathrm{min} \text {. } \\
\text { Urine output }>200 \mathrm{ml} / 24 \mathrm{~h} \text {. }\end{array}$ & $\begin{array}{l}\text { Anti-GBM disease, life threatening conditions, } \\
\text { contraindications to immunosuppression, previous } \\
\text { treatment with AZA or CPA for }>14 \text { days. }\end{array}$ \\
\hline Mauri 1985 & $\begin{array}{l}\text { Histologically proven crescentic } \mathrm{GN} \text { and rapidly } \\
\text { progressive renal impairment. }\end{array}$ & $\begin{array}{l}\text { Less than } 60 \% \text { glomerular involvement, primary } \\
\text { glomerulopathies, transplanted kidneys, SLE, HSP. }\end{array}$ \\
\hline Pusey 1991 & $\begin{array}{l}\text { Focal necrotizing GN with crescents (Wegener's } \\
\text { granulomatosis, systemic vasculitis, polyarteritis, } \\
\text { idiopathic RPGN) }\end{array}$ & $\begin{array}{l}\text { Anti-GBM disease, SLE, Henoch-Schonlein Purpura, } \\
\text { chronic GN Previously treated with IV MP, oral CPA or PE }\end{array}$ \\
\hline Rifle 1980 & New onset RPGN with > 50\% glomerular crescents. & $\begin{array}{l}\text { Goodpasture's syndrome; IgA nephropathies; SLE; } \\
\text { systemic disease. }\end{array}$ \\
\hline
\end{tabular}

ing major renal involvement. Six month results are reported in abstract form only. Primary outcome was disease remission in the absence of prednisolone therapy at 6 months. The results reported here are disease remission at 6 months on Prednisolone $10 \mathrm{mg} / \mathrm{d}$ or less. As shown in Figure 7 remission rates were similar between the two groups.

\section{Mycophenolate Mofetil for remission induction}

The use of MMF for remission induction is compared with CPA by $\mathrm{Hu}$ et al [21]. Thirty five patients with newly diagnosed ANCA associated vasculitis and moderate renal involvement were randomised to either $2 \mathrm{~g} / \mathrm{d}$ MMF or standard intravenous CPA monthly pulses for 6 months.

The primary outcome measure was remission rate at 6 months. MMF was found to be equivalent to CPA in this limited population for remission at 6 months (1 study; risk ratio 1.65 ; $95 \%$ confidence interval 0.94 to 2.90 ).
There were no data on relapse rate on either treatment. Side effects appeared similar on both agents (risk ratio 0.65 ; $95 \%$ confidence interval 0.20 to 2.14 )

\section{Intravenous immunoglobulin for treatment of persistent disease}

IVIg treatment for persistent disease has been studied by a single RCT [16]. Thirty four patients with persistent disease activity were randomised to IVIg $0.4 \mathrm{~g} / \mathrm{kg} / \mathrm{d}$ for 3 days or to identical placebo injections. The primary outcome was a response to treatment defined as a $50 \%$ reduction in BVAS (Birmingham Vasculitis Activity Score).

The use of IVIg demonstrated a therapeutic response in more patients at 3 months when compared with placebo. (1 study; risk ratio 2.33 ; $95 \%$ confidence interval 1.18 to 4.61). Benefit was not demonstrated beyond 3 months. There was no demonstrated difference in mortality or rate of relapse. 
Table 2: Interventions in the plasma exchange studies

\begin{tabular}{|c|c|c|c|}
\hline Study ID & Treatment & Control & Study outcomes \\
\hline $\begin{array}{l}\text { Cole } \\
1992\end{array}$ & $\begin{array}{l}\text { PE: at least } 10 \text { PE treatments } \\
\text { within } 16 \text { days of trial entry. } \\
\text { One plasma volume with complete } \\
\text { replacement using } 5 \% \text { albumin + } \\
\text { crystalloid. } \\
\text { Immunosuppression: as for } \\
\text { control group }\end{array}$ & $\begin{array}{l}\text { Immunosuppression } \\
\text { IV MP } 10 \mathrm{mg} / \mathrm{kg} / \mathrm{d} \text { for } 3 \text { days followed } \\
\text { by prednisone } 1.4 \mathrm{mg} / \mathrm{kg} / \mathrm{d} \text { for next } 4 \text { days } \\
\text { and then tapered to } 1 \mathrm{mg} / \mathrm{kg} / \mathrm{d} \text { over } 2 \\
\text { weeks } \\
0.35 \mathrm{mg} / \mathrm{kg} / \mathrm{d} \text { at } 1 \mathrm{month} \text { and } 0.25 \mathrm{mg} / \mathrm{kg} / \mathrm{d} \\
\text { at } 2 \mathrm{months} \text {. } \\
\text { AZA } 1.5-3.0 \mathrm{mg} / \mathrm{kg} / \mathrm{d} \text { with dose } \\
\text { adjustment } \\
\text { as necessary to ensure neutrophil count of } \\
2.0 \times 10^{9} / \mathrm{L} \text { or greater }\end{array}$ & $\begin{array}{l}\text { 1. renal pathology } \\
\text { 2. Patients on dialysis at } \\
\text { randomisation - dialysis at } 1 \text {, } \\
3,6,12 \text { months } \\
\text { 3. Renal function in patients } \\
\text { not on dialysis - } 1,3,6,12 \text { months } \\
\text { 4. Change in SCr } \\
\text { 5. Adverse events (serious infections, } \\
\text { gastrointestinal bleeding) } \\
\text { 6. Mortality }\end{array}$ \\
\hline $\begin{array}{l}\text { Jayne } \\
2007\end{array}$ & $\begin{array}{l}\text { Seven PE of } 60 \mathrm{ml} / \mathrm{kg} \text { in first } 2 \\
\text { weeks after diagnosis } \\
\text { Immunosuppression as for control } \\
\text { group }\end{array}$ & $\begin{array}{l}\text { Three pulses of } 1000 \mathrm{mg} \text { IV MP } \\
\text { followed by oral CPA and a } \\
\text { tapering regimen of prednisolone. }\end{array}$ & $\begin{array}{l}\text { 1. Mortality } \\
\text { 2. Dialysis } \\
\text { 3. Side effects } \\
\text { 4. Serum creatinine } \\
\text { at } 12 \text { months }\end{array}$ \\
\hline $\begin{array}{l}\text { Glockner } \\
1988\end{array}$ & $\begin{array}{l}\text { Nine } 50 \mathrm{ml} / \mathrm{kg} \text { PE over } 4 \text { weeks } \\
\text { replaced with 3-5\% Albumin solution. } \\
\text { Immunosuppression as for control group }\end{array}$ & $\begin{array}{l}\text { CPA } 3 \mathrm{mg} / \mathrm{kg} / \mathrm{d} \text { plus AZA } 1 \mathrm{mg} / \mathrm{kg} / \mathrm{d} \text { for } \\
1 \text { week then AZA } 2 \mathrm{mg} / \mathrm{kg} / \mathrm{d} \text {. 6-methyl } \\
\text { prednisolone } 1.5 \mathrm{mg} / \mathrm{kg} / \mathrm{d} \text { for } 14 \text { days } \\
\text { reducing in } 4 \mathrm{mg} / \mathrm{d} \text { steps to } \\
\text { maintenance } 8 \mathrm{mg} / \mathrm{d} \text {. }\end{array}$ & $\begin{array}{l}\text { 1. Mortality at } 6 \text { months } \\
\text { 2. Dialysis at } 6 \text { months } \\
\text { 3. SCr at } 4 \text { weeks, } 8 \text { weeks } \\
\text { and } 6 \text { months } \\
\text { 4. Adverse events including } \\
\text { serious infections, Gl } \\
\text { haemorrhage and anaphylaxis }\end{array}$ \\
\hline $\begin{array}{l}\text { Mauri } \\
1985\end{array}$ & $\begin{array}{l}\text { PE alternate days for } 6 \text { treatments. } \\
\text { Exchanges of at least } 3.5 \mathrm{~L} \text { replaced } \\
\text { with } 3.5 \% \text { Albumin and } 2 \text { units FFP. } \\
\text { Immunosuppression as for control group }\end{array}$ & $\begin{array}{l}\text { CPA } 2 \mathrm{mg} / \mathrm{kg} / \mathrm{d} \text { and Prednisolone } \\
1 \mathrm{mg} / \mathrm{kg} / \mathrm{d} \text {. Doses reduced to half } \\
\text { after } 8 \text { weeks. Prednisolone dose } \\
\text { tapered progressively. CPA dose } \\
\text { reduced to } 0.5 \mathrm{mg} / \mathrm{kg} / \mathrm{d} \text { after } 2 \\
\text { months then stopped after month } 4 \text {. }\end{array}$ & $\begin{array}{l}\text { 1. Mortality } \\
\text { 2. Dialysis post } \\
\text { treatment, at } 3 \\
\text { months and } 12 \text { months } \\
\text { after treatment } \\
\text { 3. SCr after treatment } \\
\text { and } 6 \text { months later }\end{array}$ \\
\hline $\begin{array}{l}\text { Pusey } \\
1991\end{array}$ & $\begin{array}{l}\text { PE: } 5 \times 4 \mathrm{~L} \text { exchanges of } 5 \% \\
\text { albumin (plasma protein fraction) } \\
\text { within first week. Two units } \\
\text { of fresh frozen plasma were given } \\
\text { at end of exchange. Total number } \\
\text { of exchanges determined by clinical } \\
\text { response. } \\
\text { Immunosuppression as for control group }\end{array}$ & $\begin{array}{l}\text { Induction therapy: } 8 \text { weeks of: } \\
1.60 \mathrm{mg} / \mathrm{d} \text { prednisolone, reduce to } 30 \mathrm{mg} / \mathrm{d} \\
\text { at week } 3 \text {, taper slowly. } \\
\text { 2. CPA } 3 \mathrm{mg} / \mathrm{kg} / \mathrm{d} \text { or } 2 \mathrm{mg} / \mathrm{kg} / \mathrm{d} \text { for those } \\
\text { over } 55 \text { years } \\
\text { 3. AZA } 1 \mathrm{mg} / \mathrm{kg} / \mathrm{d} \text { or no AZA for those } \\
\text { over } 55 \text { years } \\
\text { Maintenance therapy: CPA stopped after } 8 \\
\text { weeks in those with remission and AZA } \\
\text { increased to } 2-3 \mathrm{mg} / \mathrm{kg} / \mathrm{d} \text {, together with } \\
\text { tapering doses of prednisolone" }\end{array}$ & $\begin{array}{l}\text { 1. Improvement (fall in } \mathrm{SCr}>25 \% \\
\text { or rise in } \mathrm{CrCl}>25 \% \text {; recovery } \\
\text { of renal function in those initially } \\
\text { on dialysis) } \\
\text { 2. SCr } \\
\text { 3. Dialysis } \\
\text { 4. Death } \\
\text { 5. Adverse event }\end{array}$ \\
\hline $\begin{array}{l}\text { Rifle } \\
1980\end{array}$ & $\begin{array}{l}\text { PE. Five sessions during } 5 \text { successive } \\
\text { days, then } 3 \text { sessions/week until } \\
15 \text { days after } \mathrm{SCr} \text { reached a plateau. } \\
\text { Treatment could not exceed } 2 \text { months. } \\
1.5 \text { plasma volumes exchanged. } \\
\text { Immunosuppression as for control } \\
\text { group }\end{array}$ & $\begin{array}{l}\text { IV pulse MP }(15 \mathrm{mg} / \mathrm{kg} / \mathrm{d} \text { for } 3 \text { days, } \\
\text { tapered to } 15 \mathrm{mg} / \mathrm{d} \text { for } 3 \text { days, then } 3 \text { new } \\
\text { pulses, then } 15 \mathrm{mg} / \mathrm{d} \text { for } 7 \text { weeks. } \\
\text { CPA } 2-3 \mathrm{mg} / \mathrm{kg} / \mathrm{d} \text { for } 2 \text { months. } \\
\text { Calcium heparinate } 9 \text { days after renal } \\
\text { biopsy for the duration of the study. }\end{array}$ & $\begin{array}{l}\text { 1. Dialysis } 2,612 \text {, } \\
24 \text { months } \\
\text { 2. CrCl at 2, } 6 \text { and } 12 \text { months. } \\
\text { 3. Recovery (off dialysis) } \\
\text { according to initial SCr level } \\
\text { 4. Recovery (off dialysis) according } \\
\text { to initial \% of crescents } \\
\text { 5. Death } \\
\text { 6. Circulating immune complexes } \\
\text { 7. Pathology changes } \\
\text { 8. Adverse events (septicaemia) }\end{array}$ \\
\hline
\end{tabular}




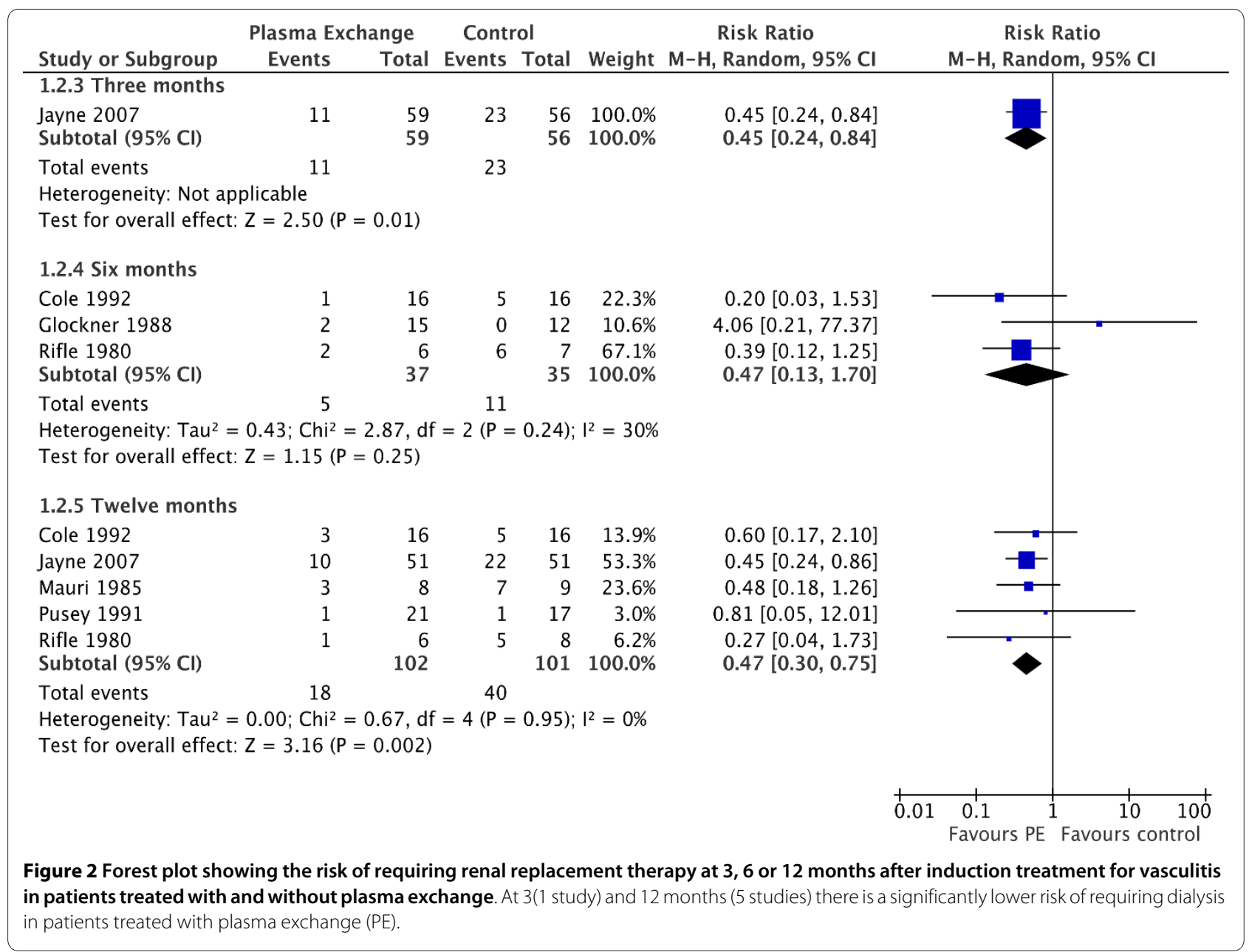

\section{Immunoadsorption and Lymphocytapheresis}

Immunoadsorption was compared to plasma exchange in a single study [38]. 44 patients were randomised to receive either immunoadsorption or plasma exchange. A median of six sessions of each treatment were delivered. Immunosuppression was otherwise the same in the groups with standard CPA and prednisolone treatment. Primary outcome was renal function and response to treatment.

There was no detected difference in efficacy between immunoadsorption and plasma exchange, as assessed by mortality, the need for dialysis and SCr.

Lymphocytapheresis is compared to 3 days of iv methylprednisolone as adjunctive therapy by Furuta et al [37]. Twenty four patients were randomised with the primary outcome being serum creatinine 4 weeks post treatment.

The use of lymphocytapheresis showed a highly significant reduction in $\mathrm{SCr}$ at four weeks (1 study; MD -2.10, 95\% CI -2.64 to -1.56$)$. Other outcomes showed a tendency to lower mortality ( 1 study; RR $0.40,95 \%$ CI 0.10 to 1.67) and fewer patients on dialysis (1 study; RR $0.33,95 \%$ CI 0.04 to 2.77 ). However, the confidence intervals for these are wide and clinical significance uncertain. No further data has been published to support this study.

\section{Maintenance Studies}

Inclusion and exclusion criteria are detailed in Table 7 and the interventions in Table 8.

\section{Azathioprine as maintenance therapy}

The use of AZA to reduce exposure to CPA is assessed by Jayne et al [39]. Patients were treated with standard induction therapy of CPA $2 \mathrm{mg} / \mathrm{kg} / \mathrm{d}$ and prednisolone 1 $\mathrm{mg} / \mathrm{kg} / \mathrm{d}$. At remission, 155 patients were randomised to either AZA $2 \mathrm{mg} / \mathrm{kg} / \mathrm{d}$ and prednisolone $10 \mathrm{mg} / \mathrm{d}$ or continued CPA $1.5 \mathrm{mg} / \mathrm{kg} / \mathrm{d}$ until 12 months, after which all patients were treated with AZA $1.5 \mathrm{mg} / \mathrm{kg} / \mathrm{d}$. Primary outcomes were relapse by 18 months and side effects of treatment.

The introduction of AZA after remission did not alter the rate of relapse at 18 months compared to the group who remained on CPA (1 study; RR 1.13, 95\% CI 0.512.5). Leukopenia was significantly less likely on AZA (1 study; RR $0.65,95 \%$ CI 0.42 to 0.99 ) though this was not 
Table 3: Inclusion criteria for the pulse versus continuous CPA studies

\begin{tabular}{|c|c|c|}
\hline Study ID & Inclusion criteria & Exclusion criteria \\
\hline Adu 1997 & $\begin{array}{l}\text { Patients } 15-70 \text { y with new-onset systemic necrotizing } \\
\text { vasculitis. } \\
\text { Wegener's granulomatosis, classical polyarteritis } \\
\text { nodosa and microscopic polyarteritis diagnosed by } \\
\text { histological or radiological evidence. }\end{array}$ & \\
\hline De Groot 2009a & $\begin{array}{l}\text { newly diagnosed Wegener's Granulomatosis, } \\
\text { microscopic polyangiitis, or renal-limited microscopic } \\
\text { polyangiitis renal involvement: at least one of: serum } \\
\text { creatinine }>150 \text { umol/l and }<500 \mu \mathrm{mol} / \mathrm{l} \text {, biopsy } \\
\text { evidence of necrotizing } \mathrm{GN} \text {, erythrocyte casts, or } \\
\text { haematuria and proteinuria, confirmatory histology or } \\
\text { ANCA positivity }\end{array}$ & $\begin{array}{l}\text { Other multi-system autoimmune disease; hepatitis B or } \\
\text { C virus or HIV infection/Serum creatinine }>500 \mu \mathrm{mol} / \mathrm{l} \text {; } \\
\text { previous cancer; pregnancy; age under } 18 \mathrm{yrs} \text { or older } \\
\text { than } 80 \text { yrs. }\end{array}$ \\
\hline Guillevin 1997 & $\begin{array}{l}\text { Age > } 15 \text { years } \\
\text { New diagnosis of systemic WG diagnosed clinically } \\
\text { based on the presence of multi-organ involvement. } \\
\text { Mono-visceral involvement representing a potential risk } \\
\text { or severe morbidity of fatality. } \\
\text { Histopathologic characterization of necrotizing } \\
\text { granulomatosis vasculitis or evidence of either } \\
\text { granulomatous inflammation and vasculitis or } \\
\text { segmental necrotizing GN }\end{array}$ & NS \\
\hline Haubitz 1998 & $\begin{array}{l}\text { New diagnoses of WG and MPA and renal involvement. } \\
\text { Biopsy performed }\end{array}$ & $\begin{array}{l}\text { Age }<18 \mathrm{y} \text {, pregnancy, HIV, malignancy, SCr }>200 \mu \mathrm{mol} / \\
\mathrm{L} \text { more than } 1 \text { year before presentation, cytotoxic drug } \\
\text { therapy for }>1 \text { week before start of study, HD for }>10 \\
\text { days before start of study. }\end{array}$ \\
\hline
\end{tabular}

reflected in an increase in infective complications in patients on CPA (1 study; RR 1.03, 95\% CI 0.51 to 2.06).

\section{MMF vs Azathioprine for maintenance treatment}

One hundred and seventy four patients with newly diagnosed AAV were randomised after successful remission induction to receive either MMF or AZA[40]. Primary outcome was time to first relapse. Time to relapse was shorter for the MMF group but this has not yet been reported in detail. The abstract published states the hazard ratio of relapse in the MMF group was 1.7 (95\% CI 1.09-2.85; $\mathrm{p}=0.03$ ) suggesting that MMF treatment results in a higher relapse rate in comparison with AZA.

\section{Azathioprine vs Methotrexate for maintenance treatment} Pagnoux et al compared AZA and MTX for maintenance treatment[23]. One hundred and twenty six patients treated to remission with standard doses of intravenous CPA and steroids were randomised to either AZA $2 \mathrm{mg} /$ $\mathrm{kg} / \mathrm{d}$ or MTX $0.3 \mathrm{mg} / \mathrm{kg} / \mathrm{wk}$ increasing by $2.5 \mathrm{mg} /$ week to a total of $25 \mathrm{mg} / \mathrm{wk}$. Primary outcome was adverse reaction causing death or leading to discontinuation of the study drug. Secondary outcomes were any adverse event, severe adverse event, relapse, relapse free survival, event free survival and quality of life.

There was no difference between the treatments for primary outcome ( 1 study; $\mathrm{RR}=0.58$ (95\% CI $0.25-1.38$ ).
Both treatments gave similar results for all the secondary outcomes.

\section{Leflunomide vs Methotrexate for maintenance treatment}

Leflunomide was compared with methotrexate for remission maintenance [22]. Fifty four patients successfully treated to remission with prednisolone and CPA, were randomised to either leflunomide or methotrexate treatment. The primary outcome was the number of relapses after 24 months on treatment.

Leflunomide was shown to be superior to methotrexate for relapse prevention in a time to event analysis in Metzler 2007. Our analysis suggests Leflunomide may be superior but does not reach statistical significance (1 study; risk ratio $0.50 ; 95 \%$ confidence interval 0.22 to 1.11). Major relapses were also reduced (risk ratio 0.15 ; 95\% confidence interval 0.02 to 1.17 ) and side effects similar on both treatments (risk ratio 2.71 ; $95 \%$ confidence interval 0.49 to 14.85 ). There were multiple methodological difficulties with this study addressed in the discussion section.

\section{Etanercept as adjunctive therapy}

The use of etanercept as adjunctive therapy in AAV was investigated by the Wegener's Granulomatosis Etanercept Trial Research Group [26]. One hundred and eighty patients with new or established WG were randomised to 
Table 4: Interventions in the pulse versus continuous CPA studies

\begin{tabular}{|c|c|c|c|}
\hline Study ID & Treatment & Control & Study outcomes \\
\hline Adu 1997 & $\begin{array}{l}\text { CPA } 15 \mathrm{mg} / \mathrm{kg} \text { and MP were given IV at } 0,2 \\
\text { and } 4 \text { weeks. The same dose was then } \\
\text { given } \\
\text { as oral pulses over a 3-day period. } \\
\text { The interval between pulses was gradually } \\
\text { increased. }\end{array}$ & $\begin{array}{l}\text { initial treatment - } 0.85 \mathrm{mg} / \mathrm{kg} \\
\text { prednisolone } \\
\text { then tapering according to a predefined } \\
\text { schedule for } 72 \text { weeks. } \\
\text { CPA } 2 \mathrm{mg} / \mathrm{kg} / \mathrm{d} \text { given until a clinical } \\
\text { decision } \\
\text { that remission had been achieved at } \\
\text { which } \\
\text { point CPA was stopped and AZA } \\
\text { commenced } \\
\text { at } 1.5 \mathrm{mg} / \mathrm{kg} / \mathrm{d}\end{array}$ & $\begin{array}{l}\text { 1. Complete and partial remission } \\
\text { 2. Relapse } \\
\text { 3. Adverse events } \\
\text { 4. Treatment failure } \\
\text { 5. Chronic dialysis }\end{array}$ \\
\hline $\begin{array}{l}\text { De Groot } \\
2009 a\end{array}$ & $\begin{array}{l}3 \text { iv pulses of CPA } 15 \mathrm{mg} / \mathrm{kg} 2 \text { weeks apart } \\
\text { followed by } 3 \text { weekly pulses ( } 15 \mathrm{mg} / \mathrm{kg} \text { iv } \\
\text { or } \\
5 \mathrm{mg} / \mathrm{kg} \text { orally for } 3 \text { days) until remission } \\
\text { then } \\
\text { for another } 3 \text { months. } \\
\text { Mac dose } 1.2 \mathrm{G} \text {. } \\
\text { Reductions for age }>60 \text { yrs and serum } \\
\text { creatinine }>300 \text { uM and for previous } \\
\text { low leukocyte nadir. }\end{array}$ & $\begin{array}{l}\text { oral CPA } 2 \mathrm{mg} / \mathrm{kg} / \mathrm{d} \text { to remission then } 1.5 \\
\mathrm{mg} / \mathrm{kg} \\
\text { for further } 3 \text { months. Max oral dose } 200 \\
\mathrm{mg} \text {. } \\
\text { Reductions for age }>60 \text { yrs and } \\
\text { leukopenia } \\
\text { Both groups received Azathioprine } 2 \mathrm{mg} / \\
\mathrm{kg} / \mathrm{d} \\
\text { orally after induction therapy until month } \\
18 \text {. } \\
\text { Prednisolone } 1 \mathrm{mg} / \mathrm{kg} \text { orally tapered to } \\
12.5 \mathrm{mg} / \mathrm{d} \\
\text { at the end of month3 and } 5 \mathrm{mg} \text { at end of } \\
\text { study }\end{array}$ & $\begin{array}{l}\text { 1. Time to Remission } \\
\text { 2. Change in renal function } \\
\text { 3. Adverse events } \\
\text { 4. Cumulative dose of CPA }\end{array}$ \\
\hline $\begin{array}{l}\text { Guillevin } \\
1997\end{array}$ & $\begin{array}{l}\text { Initial regimen: IV MP } 15 \mathrm{mg} / \mathrm{kg} / \mathrm{d} \text { for } 3 \\
\text { days. } \\
\text { IV CPA } 0.7 \mathrm{~g} / \mathrm{m}^{2} \text { day } 4 . \\
\text { Oral prednisolone } 1 \mathrm{mg} / \mathrm{kg} / \mathrm{d} \text { from day } 4 \text {. } \\
\text { IV pulse CPA: mean dose } 0.7 \mathrm{~g} / \mathrm{m}^{2} \\
\text { adjusted for } \\
\text { count and renal function, administered } \\
\text { every } \\
3 \text { weeks until complete remission and } 1 \\
\text { year thereafter. } \\
\text { Then every } 4 \text { weeks for } 4 \text { months, every } 5 \\
\text { weeks for } 4 \text { months and every } 6 \text { weeks } \\
\text { until } \\
\text { discontinuation after } 2 \text { years if treatment. } \\
\text { Adjusted up or down based on neutrophil } \\
\text { count. }\end{array}$ & $\begin{array}{l}\text { Initial regimen: IV MP } 15 \mathrm{mg} / \mathrm{kg} / \mathrm{d} \text { for } 3 \\
\text { days. } \\
\text { IV CPA } 0.7 \mathrm{~g} / \mathrm{m}^{2} \text { day } 4 . \\
\text { Oral prednisolone } 1 \mathrm{mg} / \mathrm{kg} / \mathrm{d} \text { from day } 4 \text {. } \\
\text { Oral CPA: } 2 \mathrm{mg} / \mathrm{kg} / \mathrm{d} \text { on day } 10 \text { after initial } \\
\text { CPA } \\
\text { pulse, after neutrophil nadir had been } \\
\text { reached. } \\
1 \text { year after complete remission, oral CPA } \\
\text { was } \\
\text { tapered by } 25 \% \text { every } 4 \text { months until } \\
\text { discontinuation. } \\
\text { Dose adjusted up or down based on } \\
\text { neutrophil count. }\end{array}$ & $\begin{array}{l}\text { 1. Treatment failure } \\
\text { 2. Complete remission } \\
\text { 3. Partial remission } \\
\text { 4. Relapse } \\
\text { 5. Death } \\
\text { 6. Side effects }\end{array}$ \\
\hline $\begin{array}{l}\text { Haubitz } \\
1998\end{array}$ & $\begin{array}{l}\text { Steroid regime: Days } 1-3,0.5 \mathrm{~g} \mathrm{IV} \mathrm{MP.} \\
\text { Day } 41 \mathrm{mg} / \mathrm{kg} / \mathrm{d} \text { oral prednisolone. } \\
\text { Tapered from day } 15 \text {. } \\
\text { IV Pulse CPA }-0.75 \mathrm{~g} / \mathrm{m}^{2} \text { every } 4 \text { th week. } \\
\text { If } \mathrm{CrCl}<30 \mathrm{ml} / \mathrm{min} \text {, initial dosage was } 0.5 \\
\mathrm{~g} / \mathrm{m}^{2} \\
\text { and increased to } 0.75 \mathrm{~g} / \mathrm{m}^{2} \text { provided } \\
\text { leukocyte } \\
\text { counts remained }>3000 / \mathrm{ml}\end{array}$ & $\begin{array}{l}\text { Steroid regime: Days } 1-3,0.5 \mathrm{~g} \text { IV MP. } \\
\text { Day } 41 \mathrm{mg} / \mathrm{kg} / \mathrm{d} \text { oral prednisolone. } \\
\text { Tapered from day } 15 \text {. Oral daily CPA } \\
-2 \mathrm{mg} / \mathrm{kg} / \mathrm{d} \text {. If } \mathrm{CrCl}<30 \mathrm{ml} / \mathrm{min} \text {, } \\
\text { initial dosage started at } 1.5 \mathrm{mg} / \mathrm{kg} / \mathrm{d} \text { and } \\
\text { increase } \\
\text { to } 2 \mathrm{mg} / \mathrm{kg} / \mathrm{d} \text { after } 2 \text { weeks provided } \\
\text { leukocyte } \\
\text { counts were }>3000 / \mathrm{ml} \mathrm{CPA} \mathrm{dose} \mathrm{reduced} \\
\text { in steps } \\
\text { of } 0.5 \mathrm{mg} / \mathrm{kg} \text {, unless leukocyte count was } \\
<2500 / \mathrm{ml} \text { then dose reduced by } 50 \% \text {, and } \\
\text { if } \\
\text { less than } 1500 / \mathrm{ml} \mathrm{drug} \mathrm{was} \mathrm{withheld} \\
\text { until increased to } 2500 / \mathrm{ml}\end{array}$ & $\begin{array}{l}\text { 1. Complete remission } \\
\text { 2. Partial remission } \\
\text { 3. Relapse } \\
\text { 4. Serious infection }\end{array}$ \\
\hline
\end{tabular}




\begin{tabular}{|c|c|c|c|c|c|c|c|c|}
\hline \multirow{2}{*}{$\begin{array}{l}\text { Study or Subgroup } \\
2.1 .1 \text { Three months }\end{array}$} & \multicolumn{2}{|c|}{ Pulse } & Continuous & $\begin{array}{l}\text { Ious } \\
\text { Total }\end{array}$ & \multirow[t]{2}{*}{ Weight } & \multirow[t]{2}{*}{$\begin{array}{c}\text { Risk Ratio } \\
\text { M-H, Random, } 95 \% \mathrm{Cl} \\
\end{array}$} & \multicolumn{2}{|c|}{$\begin{array}{c}\text { Risk Ratio } \\
\text { M-H, Random, } 95 \% \text { Cl }\end{array}$} \\
\hline & & & & & & & & \\
\hline Adu 1997 & 2 & 12 & 2 & 20 & $58.5 \%$ & $1.67[0.27,10.33]$ & & \\
\hline $\begin{array}{l}\text { de Groot } 2009 \text { a } \\
\text { Subtotal }(95 \% \mathrm{Cl})\end{array}$ & 1 & $\begin{array}{l}76 \\
88\end{array}$ & 4 & $\begin{array}{l}73 \\
93\end{array}$ & $\begin{array}{r}41.5 \% \\
100.0 \%\end{array}$ & $\begin{array}{l}0.24[0.03,2.10] \\
0.69[0.10,4.73]\end{array}$ & $\longleftarrow$ & \\
\hline \multicolumn{9}{|c|}{$\begin{array}{l}\text { Heterogeneity: } \text { Tau }^{2}=0.90 ; \mathrm{Chi}^{2}=1.86, \mathrm{df}=1(\mathrm{P}=0.17) ; \mathrm{I}^{2}=46 \% \\
\text { Test for overall effect: } \mathrm{Z}=0.38(\mathrm{P}=0.71)\end{array}$} \\
\hline \multicolumn{9}{|l|}{ 2.1.2 Six months } \\
\hline Adu 1997 & 2 & 12 & 3 & 20 & $39.1 \%$ & $1.11[0.22,5.73]$ & & \\
\hline $\begin{array}{l}\text { de Groot } 2009 a \\
\text { Subtotal }(95 \% \mathrm{CI})\end{array}$ & 3 & $\begin{array}{l}76 \\
88\end{array}$ & 7 & $\begin{array}{l}73 \\
93\end{array}$ & $\begin{array}{r}60.9 \% \\
100.0 \%\end{array}$ & $\begin{array}{l}0.41[0.11,1.53] \\
0.61[0.22,1.69]\end{array}$ & & \\
\hline \multicolumn{9}{|c|}{$\begin{array}{l}\text { Heterogeneity: } \mathrm{Tau}^{2}=0.00 ; \mathrm{Chi}^{2}=0.87, \mathrm{df}=1(\mathrm{P}=0.35) ; \mathrm{I}^{2}=0 \% \\
\text { Test for overall effect: } \mathrm{Z}=0.95(\mathrm{P}=0.34)\end{array}$} \\
\hline \multicolumn{9}{|l|}{ 2.1.3 One year } \\
\hline Adu 1997 & 3 & 12 & 3 & 20 & $21.8 \%$ & $1.67[0.40,6.97]$ & & \\
\hline de Groot 2009a & 5 & 76 & 9 & 73 & $40.9 \%$ & $0.53[0.19,1.52]$ & & 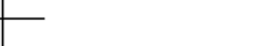 \\
\hline $\begin{array}{l}\text { Guillevin } 1997 \\
\text { Subtotal }(95 \% \mathrm{Cl})\end{array}$ & 4 & $\begin{array}{r}27 \\
115\end{array}$ & 7 & $\begin{array}{r}23 \\
116\end{array}$ & $\begin{array}{r}37.3 \% \\
100.0 \%\end{array}$ & $\begin{array}{l}0.49[0.16,1.46] \\
0.66[0.34,1.31]\end{array}$ & & 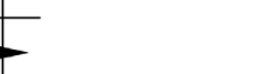 \\
\hline \multicolumn{9}{|c|}{$\begin{array}{l}\text { Heterogeneity: } \mathrm{Tau}^{2}=0.01 ; \mathrm{Chi}^{2}=2.07, \mathrm{df}=2(\mathrm{P}=0.36) ; \mathrm{I}^{2}=3 \% \\
\text { Test for overall effect: } \mathrm{Z}=1.18(\mathrm{P}=0.24)\end{array}$} \\
\hline \multicolumn{9}{|l|}{ 2.1.4 Two years } \\
\hline Adu 1997 & 4 & 12 & 3 & 20 & $27.6 \%$ & $2.22[0.60,8.28]$ & & \\
\hline Guillevin 1997 & 6 & 27 & 10 & 23 & $66.7 \%$ & $0.51[0.22,1.19]$ & & - \\
\hline $\begin{array}{l}\text { Haubitz } 1998 \\
\text { Subtotal }(95 \% \mathrm{Cl})\end{array}$ & 0 & $\begin{array}{l}22 \\
61\end{array}$ & 3 & $\begin{array}{l}25 \\
68\end{array}$ & $\begin{array}{r}5.6 \% \\
100.0 \%\end{array}$ & $\begin{array}{l}0.16[0.01,2.96] \\
0.75[0.21,2.61]\end{array}$ & & \\
\hline \multicolumn{9}{|c|}{$\begin{array}{l}\text { Heterogeneity: } \mathrm{Tau}^{2}=0.65 ; \mathrm{Chi}^{2}=4.50, \mathrm{df}=2(\mathrm{P}=0.11) ; \mathrm{I}^{2}=56 \% \\
\text { Test for overall effect: } \mathrm{Z}=0.46(\mathrm{P}=0.65)\end{array}$} \\
\hline $\begin{array}{l}\text { 2.1.5 Five years } \\
\text { Subtotal }(95 \% \mathrm{Cl})\end{array}$ & & 0 & & 0 & & Not estimable & & \\
\hline $\begin{array}{l}\text { Total events } \\
\text { Heterogeneity: Not ap } \\
\text { Test for overall effect }\end{array}$ & $\begin{array}{l}0 \\
\text { oplicable } \\
\text { Not appl }\end{array}$ & licable & 0 & & & & & \\
\hline \multicolumn{9}{|c|}{ 2.1.6 Death at final follow-up } \\
\hline Adu 1997 & 4 & 12 & 3 & 20 & $13.9 \%$ & $2.22[0.60,8.28]$ & & \\
\hline de Groot 2009a & 5 & 76 & 9 & 73 & $22.1 \%$ & $0.53[0.19,1.52]$ & & . \\
\hline Guillevin 1997 & 9 & 27 & 10 & 23 & $48.0 \%$ & $0.77[0.38,1.56]$ & & \\
\hline $\begin{array}{l}\text { Haubitz } 1998 \\
\text { Subtotal }(95 \% \mathrm{Cl})\end{array}$ & 3 & $\begin{array}{r}22 \\
137\end{array}$ & 7 & $\begin{array}{r}25 \\
141\end{array}$ & $\begin{array}{r}16.0 \% \\
100.0 \%\end{array}$ & $\begin{array}{l}0.49[0.14,1.66] \\
0.77[0.44,1.32]\end{array}$ & & \\
\hline \multirow{2}{*}{\multicolumn{9}{|c|}{$\begin{array}{l}\text { Heterogeneity: } \text { Tau }^{2}=0.05 ; \mathrm{Chi}^{2}=3.52, \mathrm{df}=3(P=0.32) ; \mathrm{I}^{2}=15 \% \\
\text { Test for overall effect: } \mathrm{Z}=0.95(P=0.34)\end{array}$}} \\
\hline & & & & & & & & \\
\hline & & & & & & & $\begin{array}{l}1 \\
0.10 .2 \quad 0.5 \\
\text { Favours pulse }\end{array}$ & 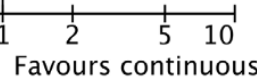 \\
\hline
\end{tabular}

receive either etanercept $25 \mathrm{mg}$ twice weekly by subcutaneous injection or placebo treatment. Immunosuppression with prednisolone and CPA for generalized or methotrexate for limited disease was also given to both groups. Primary outcome was sustained remission with a BVAS/WG of 0 for at least 6 months.

Etanercept was not shown to be effective in remission induction or relapse prevention. There was no difference 


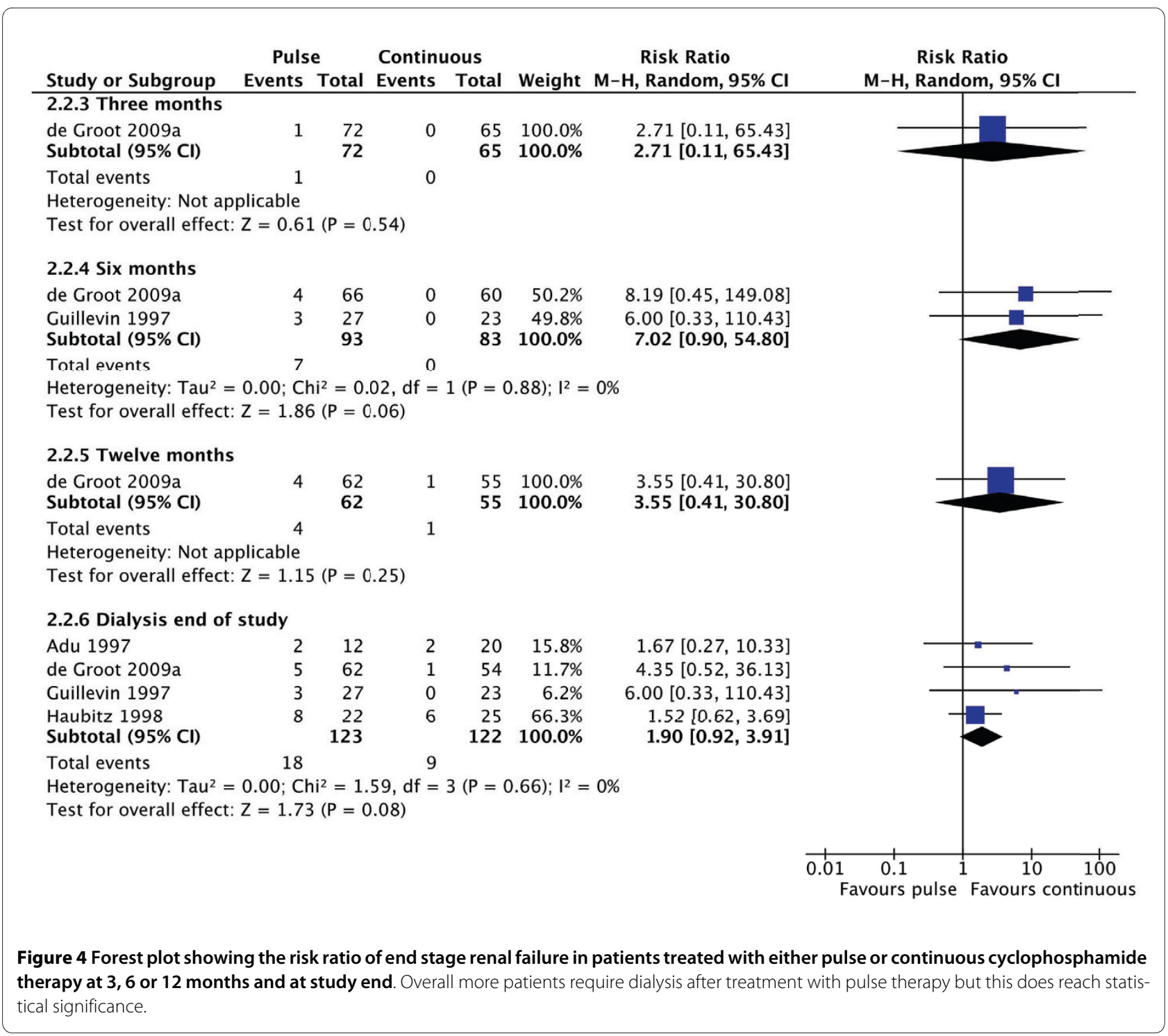

in the induction of sustained remission ( 1 study; risk ratio $0.93 ; 95 \%$ confidence interval 0.77 to 1.11 ) or relapse rate ( 1 study; risk ratio 0.93 ; $95 \%$ confidence interval 0.56 to 1.56). Moreover, the incidence of solid cancers detected during the study was higher in the etanercept arm.

\section{Co-trimoxazole for relapse prevention}

Stegeman [41] studied the efficacy of co-trimoxazole in preventing relapse of patients with ANCA associated vasculitis. Eighty patients with vasculitis and either RPGN on biopsy or otherwise meeting the Chapel Hill Consensus were randomised to co-trimoxazole $960 \mathrm{mg}$ twice daily or placebo. Primary outcomes were death, remission and relapse rate.

The reduction in the number of relapses in patients treated with co-trimoxazole was a significant result on the basis of life-table analysis, giving a reported RR of relapse of 0.40 (95\% CI 0.17 to 0.98 ). Our review, using a fixed time analysis, suggests a smaller difference between the groups with a higher probability of maintaining a remission on antibiotics ( 1 study; RR $1.28,95 \%$ CI 0.94 to 1.76) although this was not statistically significant (Figure $8)$.

\section{Risk of bias in included studies}

A summary of quality measures is shown in Table 9. Most of the studies in this review were not blinded. Only three reported blinding of patient, physicians and outcome assessors. The majority of reports do not contain adequate data to clearly indicate the risk of bias. Intention to treat analysis was performed in 10 of 18 studies and follow-up was good, ranging from 82 to $100 \%$. 


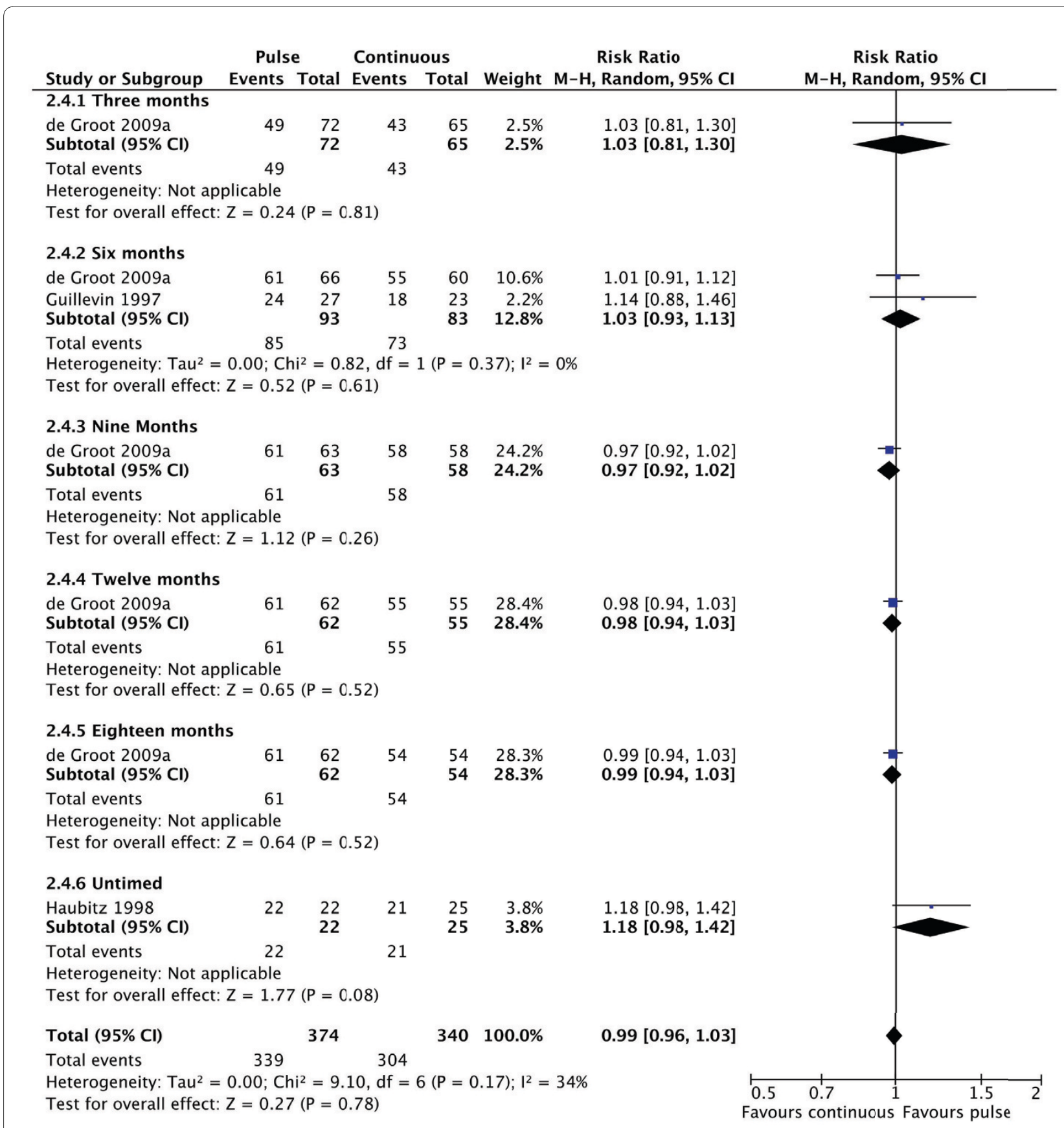

Figure 5 Forest plot showing the risk ratio of achieving remission in patients treated with pulse or continuous cyclophosphamide at various time points. There is no change in the rate of remission with pulse cyclophosphamide.

\section{Discussion}

\section{Remission Induction}

\section{Plasma exchange as adjunctive therapy}

This meta-analysis shows that plasma exchange confers a significant benefit to many patients with RPGN by reducing the risk of end stage renal failure at 12 months from diagnosis. The RR of 0.47 suggests that the number of patients requiring dialysis may be halved by this interven- tion. Previous studies have shown an effect in the most severely ill patients. A subgroup analysis in Pusey 1991 showed a benefit for patients requiring dialysis at presentation. More recently, Jayne 2007 has shown a benefit for patients with $\mathrm{SCr}$ greater than $500 \mathrm{uM}$ with ANCA associated vasculitis. The majority of patients included in these studies would meet the criteria for having severe $\mathrm{AKF}(\mathrm{SCr}>500 \mathrm{uM}$ or dialysis required at presentation). 


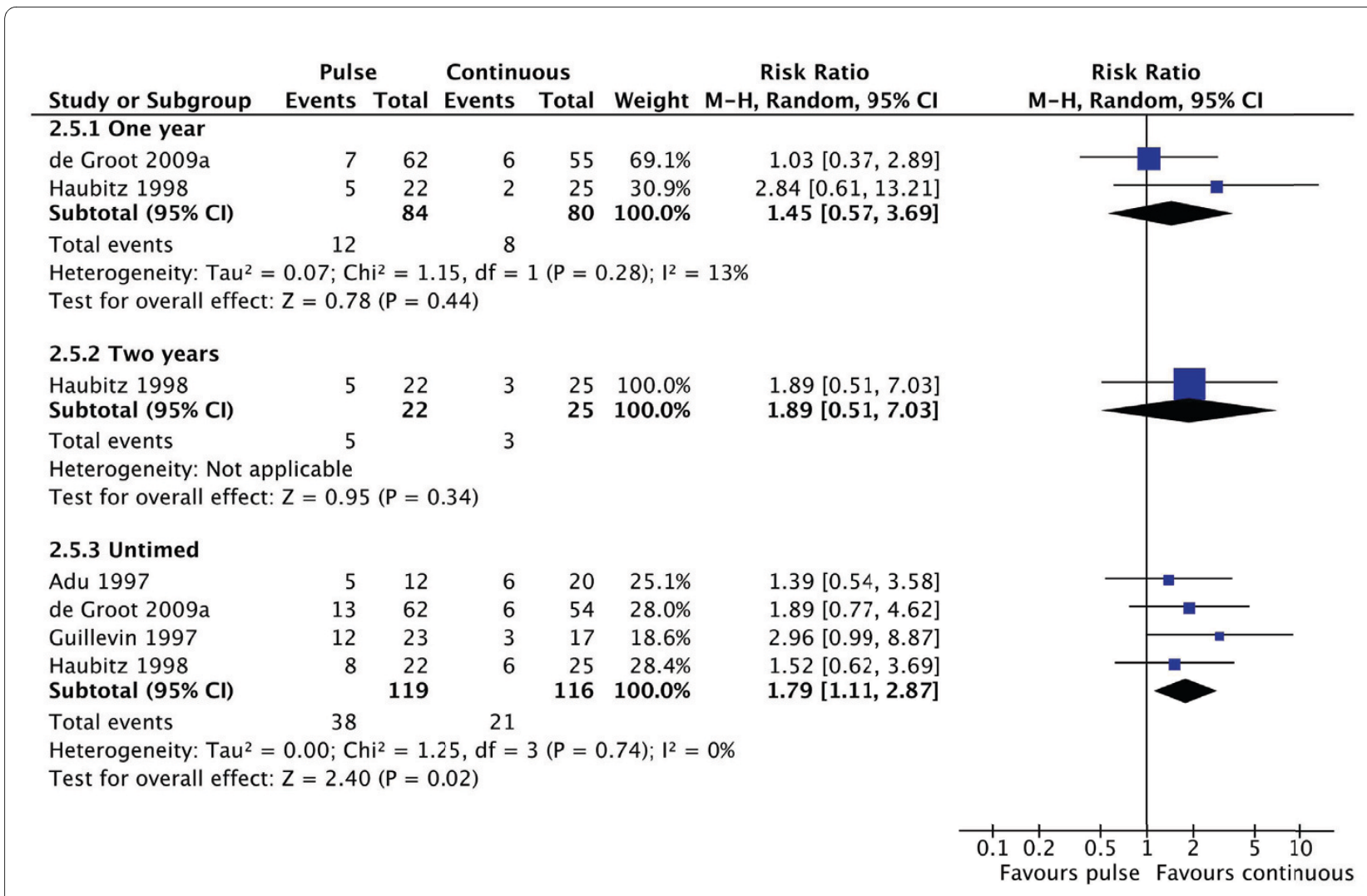

Figure 6 Forest plot showing the risk ratio of relapse in patients treated with continuous or pulse CPA at 1 and 2 years and at study end Overall continuous treatment with CPA results in a reduced rate of relapse.

It is therefore not clear whether plasma exchange has any impact in patients whose kidney failure is not severe. There was little statistical heterogeneity in all outcomes of these studies with the single exception of $\mathrm{SCr}$ at 12 months.

\section{Pulse versus continuous CPA}

This analysis strongly suggests that, although pulse treatment with cyclophosphamide is equivalent to continuous treatment for remission induction, it results in a higher rate of relapse subsequently. None of the studies in this area have been adequately powered to answer the question of relapse rate since this would require either much larger studies or significantly longer follow-up. We are therefore reliant on the results of meta-analysis to attempt to provide an answer. This answer is less than perfect since it is a meta-analysis of results at different times post treatment across studies with significantly different protocols. In spite of that, there is no evidence of heterogeneity in the outcome, suggesting that the final result is likely to be valid. Though the rates of relapse with pulse CPA treatment are perhaps discouraging, this does not invalidate this mode of treatment. Pulse therapy still delivers a significantly lower total dose of CPA. For those patients who remain in remission, they have clearly benefited in terms of risk of long term side effects.
There is a trend towards more patients requiring dialysis with the use of pulse CPA therapy. This is currently not statistically significant, but the fact remains that there were twice as many patients requiring dialysis after pulse therapy and that this effect is present in all studies. Further studies are required to clarify this question.

CPA treatment was given for three months, approximately six months, one and two years in the four relevant studies. This difference may account for the significant level of statistical heterogeneity detected in mortality and the incidence of serious infections. Pulse therapy also causes significantly more nausea but less leukopenia and serious infections. In the light of data from Jayne 2003, it would seem reasonable to suggest that continuous oral CPA should be limited to three months treatment if the patient has achieved a sustained remission with a change to AZA for maintenance therapy. The optimal regimen for CPA administration for remission induction in ANCA associated vasculitis remains unclear.

\section{Rituximab vs cyclophosphamide alone for remission induction}

The RITUXVAS and RAVE studies are two well designed studies showing that Rituximab is equivalent to CPA therapy for remission induction whilst side effects occur at a similar frequency albeit possibly in a smaller number 
Table 5: Inclusion criteria for other remission induction studies

\begin{tabular}{|c|c|}
\hline Study ID & Inclusion criteria \\
\hline Hu 2008 & $\begin{array}{l}\text { Newly diagnosed active ANCA associated vasculitis. } \\
\text { Over } 18 \text { years of age with renal involvement } \\
\text { with serum creatinine }<500 \text { uM. } \\
\text { ANCA positive or ANCA negative with confirmatory } \\
\text { renal Biopsy }\end{array}$ \\
\hline $\begin{array}{l}\text { Jones } 2008 \\
\text { (RITUXVAS) }\end{array}$ & $\begin{array}{l}\text { A new diagnosis of Wegener's Granulomatosis (WG), } \\
\text { Microscopic Polyangiitis (MP) or Renal-Limited } \\
\text { Vasculitis (RLV) } \\
\text { Renal involvement attributable to active WG, MP or } \\
\text { RLV with at least one of the following: } \\
\text { Biopsy demonstrating necrotizing glomerulonephritis } \\
\text { Red cell casts on urine microscopy or } \geq++ \text { haematuria } \\
\text { Anti-Neutrophil Cytoplasmic Antibodies (ANCA) positivity; } \\
\text { ANCA positivity requires either: } \\
\text { Proteinase } 3 \text { anti-neutrophil cytoplasmic antibody } \\
\text { (PR3-ANCA) by Enzyme-Linked Immunosorbent Assay (ELISA) } \\
\text { or a typical anti-neutrophil cytoplasmic antibody (cANCA) } \\
\text { pattern by indirect immunofluorescence (IIF), or both } \\
\text { Myeloperoxidase-anti-neutrophil cytoplasmic antibody } \\
\text { (MPO-ANCA) by ELISA. A positive peri-nuclear anti-neutrophil } \\
\text { cytoplasmic antibody (pANCA) by IIF requires } \\
\text { confirmation by MPO-ANCA ELISA. }\end{array}$ \\
\hline
\end{tabular}

Stone $2009 \quad$ Weight of at least $88 \mathrm{lbs}(40 \mathrm{~kg})$. Diagnosis of

(RAVE) Wegener's granulomatosis (WG) or microscopic polyangiitis (MPA) according to the definitions of the Chapel Hill Consensus Conference. Newly diagnosed patient of WG or MPA

OR must be experiencing a disease flare characterized by: (a) active disease with a Birmingham Vasculitis Activity Score for Wegener's granulomatosis (BVAS/WG) of 3 or greater that would normally require treatment with $C P A ; O R(b)$ disease severe enough to require treatment with CPA; OR (c) must be positive for either PR3-ANCA or MPO-ANCA at the screening. Willing to use acceptable forms of contraception for the duration of the study and for up to 1 year after stopping study medications. Willing to report pregnancies (female participants or male participants' partners) occurring at any time during the study and for up to 1 year after stopping study medications.

Parent or guardian willing to provide informed consent, if applicable.

Jayne 2000
Exclusion criteria

Cytotoxic drug treatment in 6 months prior. HBV, HCV, HIV or active CMV viral infection, acquired immune deficiency, severe renal failure with creatinine $>500$ uM or on renal replacement therapy, life-threatening organ manifestations (lung haemorrhage or CNS involvement), active TB, liver dysfunction, pregnancy or inadequate contraception if female, under age 18 or over 65 .

1. Previous cyclophosphamide, (greater than two weeks of an oral or intravenous [IV] pulse cyclophosphamide regimen)

2. Co-existence of another multi-system autoimmune disease, e.g. SLE, Churg Strauss syndrome, Henoch Schonlein purpura, rheumatoid vasculitis, essential mixed cryoglobulinaemia, anti-glomerular basement membrane antibody positivity

3. Hepatitis $B$ antigen positive or hepatitis $C$ antibody positive 4. Known HIV positive (HIV testing will not be a requirement for this trial)

5. Previous malignancy (usually exclude unless agreed with trial co-ordinator)

6. Pregnancy, breast feeding or inadequate contraception if female

7. Allergy to a study medication

8. Live vaccine within last four weeks

Churg-Strauss Syndrome

Limited disease that would not normally be treated with CPA. Mechanical ventilation because of alveolar haemorrhage History of severe allergic reactions to human or chimeric monoclonal antibodies.

Active systemic infection.

Deep-space infection, such as osteomyelitis, septic arthritis, or pneumonia complicated by pleural cavity or lung abscess, within 6 months prior to study entry.

History of or current hepatitis B or C infection. HIV infected. Acute or chronic liver disease. History of or active cancer diagnosed within the last 5 years. History of anti-glomerular basement membrane (anti-GBM) disease.

Other uncontrolled disease, including drug and alcohol abuse.

Pregnancy or breastfeeding.

IVIg in previous 3 months history of anaphylaxis to matched blood products selective $\lg$ A deficiency RPGN (20\% rise in creatinine in 2 weeks) or pulmonary haemorrhage.

None Stated

HIV, hepatitis A, B or C virus, severe congestive heart failure, malignancy, septicaemia. 
Table 6: Interventions in other remission induction studies

\begin{tabular}{ll}
\hline Study ID & Treatment \\
\hline Hu 2008 & $\begin{array}{l}\text { MMF } 2 \mathrm{G} / \mathrm{d}(1.5 \mathrm{G} \text { if weight }<50 \mathrm{~kg} \text { ) for } 6 \\
\text { months Immunosuppression as for } \\
\text { control group }\end{array}$ \\
& \\
Jones 2008 & $\begin{array}{l}\text { Rituximab, } 375 \mathrm{mg} / \mathrm{m}^{2} \mathrm{IV} \text { once a week for } \\
\text { (RITUXVAS) }\end{array}$ \\
$\begin{array}{l}\text { 4 weeks (i.e. } 4 \text { doses total), with } 2 \text { doses } \\
\text { of cyclophosphamide } 15 \mathrm{mg} / \mathrm{kg}, 2 \\
\text { weeks apart given with the } 1 \text { st and 3rd } \\
\text { rituximab dose. } \\
\text { All patients received } 1 \mathrm{~g} \text { IV } \\
\text { methylprednisolone, then same daily } \\
\text { oral corticosteroid regimen. }\end{array}$
\end{tabular}

Stone 2009 rituximab $\left(375 \mathrm{mg} / \mathrm{m}^{2}\right)$ infusions once (RAVE)

Jayne 2000

$$
\text { IVIg } 0.4 \mathrm{G} / \mathrm{kg} / \mathrm{d} \text { for } 5 \text { days }
$$

\section{Control}

Intravenous CPA for 6 months as 0.75 $1.0 \mathrm{G} / \mathrm{m}^{2}$, modified depending on WCC nadir Iv MethylPrednisolone $0.5 \mathrm{G}$ daily for three days both groups followed by oral prednisolone at $0.6-0.8 \mathrm{mg} / \mathrm{kg} / \mathrm{d}$ for 4 weeks tapered by $5 \mathrm{mg} / \mathrm{wk}$ to $10 \mathrm{mg} / \mathrm{d}$

CPA $15 \mathrm{mg} / \mathrm{kg}$ for 3-6 months (6-10 doses total)

IV for remission induction. AZA for remission maintenance.

All patients received $1 \mathrm{~g} \mathrm{IV}$ methylprednisolone, then same daily oral corticosteroid regimen.

Study outcomes

1. Remission rate at 6 months. Defined as no clinical signs of vasculitis, improved or stable renal function, no active urinary sediment and BVAS score 0 . 2. Changes in renal function, side effects

1. Primary

i) Sustained remission (BVAS $=0$ at 6 months and sustained for 6 months). ii) Severe adverse events at 2 years. 2. Secondary

a. Efficacy Response rate at 6 weeks (BVAS $<50 \%$ baseline) Remission at 6 months (BVAS $=0$ for 2 months by 6 months) Time to remission (BVAS $=0$ ) Relapses (all relapses and major/minor) BVAS area under the curve Change in GFR Change in SF-36 Change in VDI b. Safety Severe adverse events at 6 weeks and 6 months. All adverse events. Death. Prednisolone cumulative dose. CPA cumulative dose 3 . Tertiary.

Human anti-chimeric antibody testing Correlation of $B$ cells with disease activity Change in ANCA and disease activity Histopathology predictors of outcome.

Primary outcome measures

rituximab placebo infusions once weekly for 4 weeks and CPA daily for 3 to 6 months Remission Maintenance: discontinue CPA and start AZA daily until Month 18.

Both groups: IV MP 1-3 G then Prednisolone $1 \mathrm{mg} / \mathrm{kg} / \mathrm{d}$ reduced to 40 mg month 1, tapered to discontinue at the end of month 6 Prednisolone $1 \mathrm{mg} / \mathrm{kg} / \mathrm{d}$ reduced to 40 mg month 1 , tapered to discontinue at the end of month 6
Placebo (identical injections) Complete remission during the first 6 months after randomisation Secondary outcome measures Adverse events
Furuta 1998 Three 1 hour sessions of lymphocytapheresis on alternate days in each of three consecutive weeks. Immunosuppression as for control group

Stegmayr 1999 Immunoadsorption: At least 2 plasma volumes were removed. Median of 6 sessions. Immunosuppression as for control group
$1 \mathrm{G} \mathrm{MP}$ iv for 3 consecutive days in each of three consecutive weeks. BOTH GROUPS: Prednisolone $20 \mathrm{mg} /$ day and CPA $50 \mathrm{mg} /$ day.

$3 \mathrm{PE}$ in first 5 days of at least 1 plasma volume.

$4 \%$ Albumin as replacement. Median of six sessions.

All patients received immunosuppression with pulse MP and oral or iv CPA $2 \mathrm{mg} / \mathrm{kg} /$ day. CPA continued for 8 weeks or longer if ANCA positive.
Primary outcome: treatment response. BVAS reduction of $50 \%$ between entry and 3 months Secondary outcomes: fall in BVAS, CRP and ANCA, relapse frequency between 3 and 12 months, reduction in immunosuppressive drug doses and adverse effect

1. $\mathrm{SCr} 4$ weeks post treatment 2. Mortality

1. $\mathrm{CrCl}$ and $\mathrm{SCr}$

2. Responder $(\mathrm{CrCl}$ improved by at least $20 \mathrm{ml} / \mathrm{min}$ or patient could leave dialysis)

3. Adverse events

4. Dialysis

5. Death 


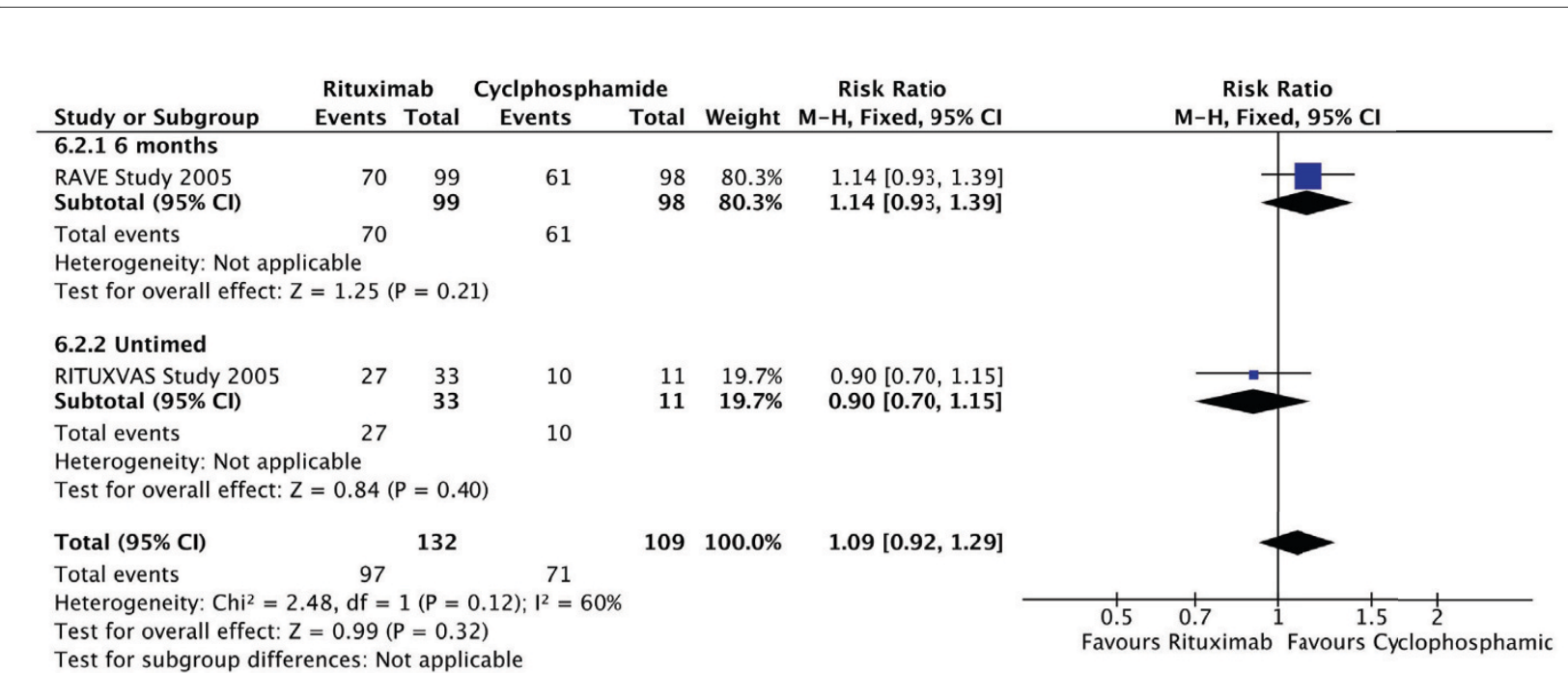

Figure 7 Forest plot showing the risk ratio for induction of remission in patients treated with or without rituximab at 6 months and at study end. There is no significant difference between the groups.

of patients with rituximab. Given recent papers suggesting that the side effects of cyclophosphamide are more common than previously thought[42], the threshold for rituximab use is likely to fall, in spite of its expense.

\section{Mycophenolate for remission induction}

The data currently available on this question remain sparse. The study by $\mathrm{Hu}$ et al is encouraging in that it does not show a reduction in remission induction with MMF. The next question is the subsequent relapse rate after MMF use for induction and this has not so far been addressed. If the relapse rate is particularly high, MMF may simply turn out to be an expensive prelude to CPA. The population in this trial is significantly different from those in other studies, most obviously in the proportion of patients with MPO-ANCA and microscopic polyangiitis at $87 \%$. This is significantly different from that reported from Europe where the majority of patients are PR3-ANCA positive. The remission rate is also lower than that achieved in similar studies from Europe with only $44 \%$ of patients achieving remission as opposed to over $90 \%$ (Jayne 2003). The external validity of the trial and wider applicability of its results remain to be established.

\section{Intravenous immunoglobulin use for refractory vasculitis}

The single RCT in this area suggests a short term benefit lasting up to 3 months. The treatment can be viewed as a therapy available to help induce remission but has little bearing on the longer term problem of remission maintenance.

\section{Lymphocytapheresis and immunoadsorption}

This novel treatment described by Furuta 1998 gives some benefit when compared with three weeks of intravenous pulse methylprednisolone with a significantly lower $\mathrm{SCr}$ in treated patients. Considering the lack of a comparison with plasma exchange and the recent data suggesting the use of plasma exchange is superior to pulse methylprednisolone, there is currently no compelling reason to consider using this therapy. Immunoadsorption, similarly, appears to have no benefit over the use of plasma exchange.

\section{Maintenance Therapy \\ Azathioprine vs CPA as maintenance therapy}

The use of AZA as maintenance therapy after an initial three month treatment with CPA is strongly supported by the data from Jayne 2003. The number of relapses on AZA is similar to CPA with fewer episodes of leukopenia and similar numbers of infections. As well as the data on reduced leukopenia, the reduction in total dose of CPA is presumed to reduce longer term side effects from CPA such as infertility and neoplasia.

\section{Leflunomide or Methotrexate for maintenance therapy}

The single study of Leflunomide suggests that this may be an appropriate treatment for patients who are intolerant of Azathioprine. There are problems with interpretation and extrapolation from this study. The dose of methotrexate was increased very slowly. Many commentators felt this to be an inadequate dose, potentially causing the higher relapse rate and reflecting poorly the potential of methotrexate in this area. There were also a high number of adverse events in the leflunomide arm. The study does however give some data on the use of Leflunomide and grounds for its clinical use. Final conclusions are difficult to draw. Further study of leflunomide is warranted as induction therapy and in comparison to Azathioprine as maintenance therapy. 
Table 7: Inclusion Criteria for studies of maintenance treatment

\begin{tabular}{|c|c|c|}
\hline Study ID & Inclusion criteria & Exclusion criteria \\
\hline Jayne 2003 & $\begin{array}{l}\text { Diagnosis of WG, MPA or renal limited vasculitis. } \\
\text { Renal involvement, other threatened loss of function of vital } \\
\text { organ, or both. } \\
\text { ANCA positivity. ANCA negative patients enrolled with biopsy } \\
\text { evidence of vasculitis. }\end{array}$ & $\begin{array}{l}\text { Cytotoxic drug in previous year. } \\
\text { Other multi-system autoimmune disease, hepatitis B } \\
\text { antigenaemia, hepatitis C, HIV infection, cancer, pregnancy. } \\
\text { Age: }<18 \text { or }>75 \text { years } \mathrm{SCr}>500 \mu \mathrm{mol} / \mathrm{L}\end{array}$ \\
\hline Stegeman 1996 & $\begin{array}{l}\text { RPGN on biopsy plus lung disease compatible with WG. } \\
\text { Disease limited to respiratory tract. } \\
\text { Meeting Chapel Hill consensus but not criteria } 1\end{array}$ & $\begin{array}{l}\text { Previous reactions to co-trimoxazole. } \\
\text { GFR }<30 \mathrm{ml} / \mathrm{min} \\
\text { Long term treatment with antibiotics }\end{array}$ \\
\hline Metzler 2007 & $\begin{array}{l}18 \text { to } 75 \text { years of age. } \\
\text { Diagnosis of generalized WG } \\
\text { Successful induction therapy with prednisolone and CPA. }\end{array}$ & $\begin{array}{l}\text { Bone marrow insufficiency (leukopenia }<4000 / \mu \mathrm{l}, \mathrm{Hb}<10 \mathrm{~g} / \\
\mathrm{dl} \text {, thrombocytopaenia }>100,000 / \mu \mathrm{l}) \text { serum creatinine }>1.3 \\
\mathrm{mg} / \mathrm{dl}(115 \mathrm{uM}) \text {, malignancies, hepatitis B or C or HIV } \\
\text { positivity, pregnancy or breast feeding, inadequate } \\
\text { contraception, chronic liver disease or alcohol abuse, active } \\
\text { gastric ulcer, lack of compliance, further coexisting } \\
\text { autoimmune diseases or treatments interfering with the } \\
\text { MTX/LEF medication. }\end{array}$ \\
\hline WGET 2005 & $\begin{array}{l}\text { Patients with BVAS/WG score of } 3 \text { or more. } \\
\text { Stratified to severe (life-threatening manifestations including } \\
\text { RPGN, alveolar haemorrhage or neuropathy) or limited (skin, } \\
\text { joints, sinus or mild renal abnormalities). } \\
\text { At least } 2 \text { of } 5 \text { modified criteria of the American College of } \\
\text { Rheumatology for classification of Wegener's } \\
\text { Granulomatosis. } \\
\text { Either new or established disease }\end{array}$ & None stated \\
\hline Pagnoux 2008 & $\begin{array}{l}\text { Patients over } 18 \text { years old. Newly diagnosed Wegener's } \\
\text { Granulomatosis and Microscopic Polyangiitis. Successful } \\
\text { remission induction. }\end{array}$ & $\begin{array}{l}\text { use of steroids for more than } 1 \text { months prior to CPA therapy, } \\
\text { co-existence of another systemic disease, cancer (unless in } \\
\text { remission for more than } 3 \text { years), HIV, Hep B or C virus } \\
\text { infection, contraindication to study drugs, pregnancy, } \\
\text { absence of contraception in pre-menopausal women, mental } \\
\text { or physical disabilities abrogating ability to consent. Patients } \\
\text { not entering remission were not randomised. }\end{array}$ \\
\hline $\begin{array}{l}\text { Hiemstra } 2009 \\
\text { (IMPROVE) }\end{array}$ & $\begin{array}{l}\text { Newly diagnosed patients with WG, MPA or renal-limited } \\
\text { vasculitis. } \\
\text { ANCA positivity. } \\
\text { Age } 18 \text { to } 75 \text { years }\end{array}$ & $\begin{array}{l}\text { Any cytotoxic drug within previous year. } \\
\text { Co-existence of another systemic autoimmune disease, e.g. } \\
\text { SLE. Hepatitis B or Hepatitis C infection HIV positivity. } \\
\text { Failure to achieve remission after } 6 \text { months of CPA therapy. } \\
\text { Failure to control progressive disease with induction } \\
\text { protocol. } \\
\text { Malignancy. } \\
\text { Pregnancy or inadequate contraception. } \\
\text { End stage renal failure unless active extra-renal disease } \\
\text { requires treatment Inability for informed consent }\end{array}$ \\
\hline
\end{tabular}

\section{Azathioprine vs Methotrexate for maintenance therapy}

This study showed that the safety and efficacy profiles of Methotrexate and AZA are comparable. This data clearly places methotrexate as a better maintenance agent that MMF since it gave similar relapse rates to AZA. The dosing regimen for MTX in this study was superior to that in the Leflunomide/Methotrexate study since the rate of rise in dose was faster and the final dose higher.

\section{Antibiotics for maintenance of remission}

The use of co-trimoxazole to maintain remission was examined by Stegeman 1996. This showed a benefit in reducing the risk of relapse but not on other outcomes.
Analysis in the paper by life table analysis showed this result to be statistically significant. On our analysis using fixed time analysis, it is not statistically significant $(\mathrm{P}=$ 0.12 ). Relapses detected in the study were mainly respiratory in nature but $11 / 23$ patients with a relapse also had progressive glomerulonephritis.

\section{Etanercept use}

The stated aim of the single study into the use of etanercept in systemic vasculitis was to demonstrate that the relapse rate would be reduced. The study failed to show this and also suggested an increase in the incidence of malignancy in treated patients. There is currently no 
Table 8: Interventions for studies of maintenance treatment

\begin{tabular}{|c|c|c|c|}
\hline Study ID & Treatment & Control & Study Outcomes \\
\hline $\begin{array}{l}\text { Hiemstra } 2009 \\
\text { (IMPROVE) }\end{array}$ & $\begin{array}{l}\text { Mycophenolate mofetil } 2 \mathrm{G} / \mathrm{d} \text { for } 42 \\
\text { months }\end{array}$ & azathioprine $2 \mathrm{mg} / \mathrm{kg} / \mathrm{d}$ for 42 months & $\begin{array}{l}\text { 1. Time to first relapse } \\
\text { 2. relapse rate } \\
\text { 3. Rate of side-effects and intolerance } \\
\text { 4. cumulative doses (AZA, CS, MMF), } \\
\text { 5. AUC for BVAS, SF-36 or VDI } \\
\text { 6. Evolution of titres of ANCA and } \\
\text { CRP }\end{array}$ \\
\hline Pagnoux 2008 & $\begin{array}{l}\text { All patients received identical } \\
\text { remission induction therapy. Pulse MP } \\
15 \mathrm{mg} / \mathrm{kg} \text { for } 3 \text { days. Oral prednisolone } \\
1 \mathrm{mg} / \mathrm{kg} / \mathrm{d} \text { for } 3 \text { weeks, tapered to } 12.5 \\
\mathrm{mg} \text { at } 6 \text { months. Pulse CPA } 0.6 \mathrm{G} / \mathrm{m}^{2}, 3 \\
\text { doses at } 2 \text { week intervals then every } 3 \\
\text { weeks until remission, } 3 \text { further } \\
\text { consolidation doses at } 3 \text { week intervals. } \\
\text { Azathioprine } 2 \mathrm{mg} / \mathrm{kg} / \mathrm{d} \text { Maintenance } \\
\text { therapy continued for } 12 \text { months then } \\
\text { withdrawn over } 3 \text { months. }\end{array}$ & $\begin{array}{l}\text { Methotrexate } 0.3 \mathrm{mg} / \mathrm{kg} / \text { week, } \\
\text { increasing every week by } 2.5 \mathrm{mg} \text { to } 25 \\
\text { mg/week. Folinic Acid } 25 \mathrm{mg} \text { or Folic } \\
\text { Acid } 5 \mathrm{mg} \text { given } 48 \text { hours after } \\
\text { Methotrexate. } \\
\text { Maintenance therapy continued for } 12 \\
\text { months then withdrawn over } 3 \\
\text { months. Trimethoprim- } \\
\text { sulfamethoxazole } 1600 / 320 \text { daily } \\
\text { recommended for WG patients for } \\
\text { additional } 2 \text { years. }\end{array}$ & $\begin{array}{l}\text { Primary end point: } \\
\text { 1. adverse reaction causing death or } \\
\text { leading to discontinuation of the study } \\
\text { drug. } \\
\text { Secondary end points: } \\
\text { 2. any adverse event, } \\
\text { 3. severe adverse event, } \\
\text { 4. relapse, } \\
\text { 5. relapse free survival, } \\
\text { 6. event free survival, } \\
\text { 7. quality of life. }\end{array}$ \\
\hline
\end{tabular}

WGET $2005 \quad$ Etanercept 25 mg twice weekly by subcutaneous injection Immunosuppression as for control group.
Metzler 2007 Leflunomide. Loading dose of $100 \mathrm{mg} /$ $\mathrm{d}$ for 3 days, followed by $20 \mathrm{mg} / \mathrm{d}$ from day 4 to end of week 4 . Then increased to $30 \mathrm{mg} / \mathrm{d}$ thereafter.

Prednisolone as per methotrexate group.

\begin{tabular}{|c|c|}
\hline Stegeman 1996 & Co-trimoxazole $960 \mathrm{mg}$ twice daily \\
\hline Jayne 2003 & $\begin{array}{l}\text { CPA } 1.5 \mathrm{mg} / \mathrm{kg} / \mathrm{d} \text { from remission. } \\
\text { Switched to AZA } 1.5 \mathrm{mg} / \mathrm{kg} / \mathrm{d} 12 \\
\text { months after study entry. } \\
\text { Immunosuppression as for control } \\
\text { group. }\end{array}$ \\
\hline
\end{tabular}

Twice weekly placebo injection Severe disease: Cyclophosphamide 2 $\mathrm{mg} / \mathrm{kg} / \mathrm{d}$.

Replaced with methotrexate if in remission at 3 to 6 months. Limited disease: Methotrexate 0.25 $\mathrm{mg} / \mathrm{kg} /$ week to maximum of $25 \mathrm{mg} /$ week. 12 months after remission, MTX dose cut by $2.5 \mathrm{mg}$ each month. Prednisolone was given to patients with severe and limited disease starting at 0.5 to $1.0 \mathrm{mg} / \mathrm{kg} / \mathrm{d}$. Tapered to $0 \mathrm{mg}$ at 6 months if no relapse.

Patients in remission with creatinine > $2 \mathrm{mg} / \mathrm{dl}$ received Azathioprine $2 \mathrm{mg} /$ $\mathrm{kg} / \mathrm{d}$, decreased after 12 months in remission by $25 \mathrm{mg}$ each month.

Methotrexate: $7.5 \mathrm{mg} /$ week weeks 1 to 4. $15 \mathrm{mg} /$ week for weeks 5 to $8.20 \mathrm{mg} /$ week after week 8 .

Prednisolone allowed at a dose of 10 $\mathrm{mg} / \mathrm{d}$ or less.

In the absence of disease activity, the dose was tapered by $2.5 \mathrm{mg} / \mathrm{month}$ to $5 \mathrm{mg}$, then by $1 \mathrm{mg} /$ month.

Placebo

After remission induction, AZA 2 mg/ $\mathrm{kg} / \mathrm{d}$ with Prednisolone $10 \mathrm{mg} / \mathrm{d}$. BOTH GROUPS: Remission induction with oral CPA $2 \mathrm{mg} / \mathrm{kg} / \mathrm{d}$ and prednisolone $1 \mathrm{mg} / \mathrm{kg} / \mathrm{d}$ tapered to $0.25 \mathrm{mg} / \mathrm{kg} / \mathrm{d}$ by 12 weeks. From 12 months both groups received AZA 1.5 $\mathrm{mg} / \mathrm{kg} / \mathrm{d}$ and prednisolone $7.5 \mathrm{mg} / \mathrm{d}$.
1. Sustained remission. BVAS/WG score 0 for at least 6 months.

2. Number and rate of flares during treatment, percentage of patients with sustained low level of disease activity (BVAS/WG $<3$ for at least 6 months), percentage of patients with a remission, cumulative area under the curve for the BVAS/WG, adverse events, quality of life.

1. Number of major and minor relapses. 2. Disease Extent Index (DEI), BVAS, patient self assessment of quality of life (SF-36), cANCA titre, ESR, CRP.

1. Death

2. Remission

1. Relapse by 18 months

2. Side effects including leucopenia and infections 


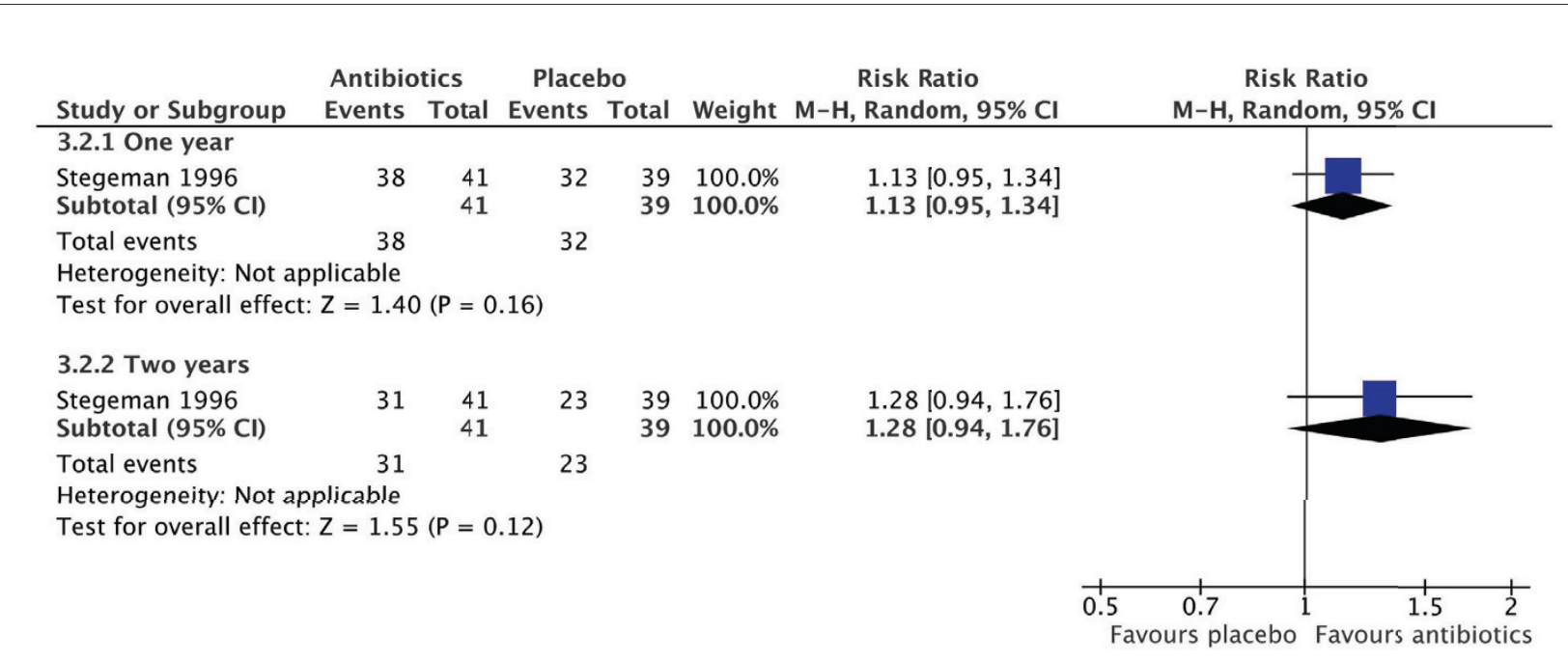

Figure 8 Forest plot of risk ratio for relapse in patients treated with or without co-trimoxazole at $\mathbf{1}$ and $\mathbf{2}$ years. There is no statistically significant difference between the groups.

RCT data on the use of infliximab or other anti-TNF agents. There is some possibility that alternative agents may produce significantly different outcomes since their mechanism of action is distinct from that of etanercept. At this point in time there is no randomised controlled trial data supporting their use.

\section{Comparison with other reviews}

Two previous reviews have covered some of the subjects addressed in this review. Bosch 2007 provides a broad review of the treatment of ANCA associated vasculitis [43]. This includes patients with localized disease and those without renal vasculitis. They include a large number of uncontrolled studies. There was no attempt at meta-analysis. In the area of severe vasculitis with kidney involvement, there is a brief summary of the randomised trial data as included in this review. Their conclusions are similar to ours. de Groot 2001 is a review of the data relating to the use of pulse or continuous CPA for induction of remission of ANCA-associated vasculitis and includes a meta-analysis of the randomised controlled trial data [13]. As such, it performs a similar metaanalysis to ours in this area. There are, however, a number of differences. de Groot 2001 has utilised all the data from the Adu study. We have extracted the data only for patients without polyarteritis nodosa and with more than $0 \%$ glomerular involvement. This accounts for some of the differences but not all. de Groot 2001 reports that treatment failure is more likely with continuous treatment with CPA. There are also some differences in results for relapse rate. Our results show that continuous treatment is significantly better at preventing relapse. We have studied relapse as related to the initial number of patients whereas de Groot 2001 have recorded relapses as related to achieved remissions. With the higher treatment failure figures in the continuous arm, de Groot 2001 does not show a significant difference in the overall relapse rate.

As stated in the introduction, a version of this review has previously been published in the Cochrane Library[19]. This is an updated version containing the latest RCT data in several areas not previously published as part of this review. Most significant is the new data on pulse versus continuous cyclophosphamide discussed above. Also included here for the first time are the data on MMF, leflunomide, IVIG, rituximab and etanercept.

\section{Authors' Conclusions Implications for practice}

Plasma exchange is effective in patients with severe ARF secondary to vasculitis. On current data, the use of pulse CPA results in an increased risk of relapse when compared to continuous use but a reduced total CPA dose. The use of co-trimoxazole is not supported for prevention of relapse of vasculitis. AZA and MTX are effective as maintenance therapy once remission has been achieved. Whilst there is evidence for the use of MMF as an induction agent, the wider applicability of the single study is questionable. The use of MMF in remission maintenance should be third line after failure of other agents such as AZA and MTX. Etanercept is not recommended for use in vasculitis. Leflunomide may be useful as maintenance therapy but requires further evaluation. IVIG is useful but only as a short term measure.

\section{Implications for research}

Further research is required to address the use of plasma exchange in patients with $\mathrm{SCr}<500 \mathrm{uM}$ at presentation. 
Table 9: Summary of quality measures of included studies

\begin{tabular}{|c|c|c|c|c|c|c|c|c|}
\hline Study ID & Randomisation method & $\begin{array}{l}\text { Allocation } \\
\text { concealment }\end{array}$ & $\begin{array}{l}\text { Blinding: } \\
\text { Participants }\end{array}$ & $\begin{array}{l}\text { Blinding: } \\
\text { Investigators }\end{array}$ & $\begin{array}{l}\text { Blinding: } \\
\text { Outcome } \\
\text { assessors }\end{array}$ & $\begin{array}{l}\text { Blinding: } \\
\text { Data assessors }\end{array}$ & ITT & $\begin{array}{c}\% \\
\text { Follow-up }\end{array}$ \\
\hline Adu 1997 & $\begin{array}{l}\text { Computer generated } \\
\text { random numbers. } \\
\text { Stratified for renal function }\end{array}$ & $A$ & No & No & No & No & Yes & 100 \\
\hline Cole 1992 & $\begin{array}{l}\text { computer-generated } \\
\text { random numbers after } \\
\text { informed consent } \\
\text { Stratified by urine volume } \\
(<400 \mathrm{ml} / 24 \mathrm{~h}), \text { need for } \\
\text { dialysis and }>50 \% \\
\text { glomeruli sclerosed }\end{array}$ & B & No & No & Yes & No & No & 97 \\
\hline $\begin{array}{l}\text { De Groot } \\
\text { 2009a }\end{array}$ & $\begin{array}{l}\text { computer generated, } \\
\text { performed centrally by } \\
\text { permuted blocks of } 4 \text {, } \\
\text { stratified by country and } \\
\text { disease. assigned randomly } \\
1: 1 \text { to treatments. }\end{array}$ & A & No & No & No & Yes & Yes & 83 \\
\hline Furuta 1998 & NS & B & No & No & NS & NS & Yes & 100 \\
\hline Jayne 2007 & NS & B & No & No & NS & NS & Yes & 100 \\
\hline $\begin{array}{l}\text { Glockner } \\
1988\end{array}$ & $\begin{array}{l}\text { By telephone with } \\
\text { statistician }\end{array}$ & B & No & No & NS & NS & No & 84 \\
\hline $\begin{array}{l}\text { Guillevin } \\
1997\end{array}$ & NS & B & No & No & NS & NS & No & 93 \\
\hline Haubitz 1998 & Stratified at diagnosis & B & No & No & NS & NS & No & 84 \\
\hline Jayne 2007 & $\begin{array}{l}\text { Centrally, block design. } \\
\text { Stratified by diagnosis }\end{array}$ & $A$ & NS & NS & NS & Yes & Yes & 100 \\
\hline Mauri 1985 & NS & B & No & No & NS & NS & Yes & 100 \\
\hline Pusey 1991 & $\begin{array}{l}\text { Stratified for severity } \\
\text { according to renal function } \\
\text { by random numbers }\end{array}$ & B & No & No & NS & NS & No & 92 \\
\hline Rifle 1980 & NS & B & No & No & NS & NS & No & 82 \\
\hline $\begin{array}{l}\text { Stegeman } \\
1996\end{array}$ & $\begin{array}{l}\text { Stratified by group and } \\
\text { then randomized }\end{array}$ & B & Yes & Yes & Yes & NS & No & 99 \\
\hline $\begin{array}{l}\text { Stegmayr } \\
1999\end{array}$ & NS & B & No & No & NS & NS & No & 83 \\
\hline Hu 2008 & Not stated & B & No & No & No & NS & Yes & 89 \\
\hline Jayne 2000 & Central & A & Yes & Yes & Yes & NS & Yes & 100 \\
\hline Metzler 2007 & Central & A & No & No & No & No & Yes & 96 \\
\hline WGET 2000 & $\begin{array}{l}\text { Stratified by disease } \\
\text { severity and by centre }\end{array}$ & A & Yes & Yes & Yes & NS & Yes & 97 \\
\hline $\begin{array}{l}\text { Pagnoux } \\
2008\end{array}$ & Permuted blocks of six & A & No & No & No & NS & Yes & 100 \\
\hline $\begin{array}{l}\text { Hiemstra } \\
2009\end{array}$ & NS & NS & No & No & NS & NS & Yes & NS \\
\hline RAVE & NS & NS & Yes & Yes & NS & NS & NS & NS \\
\hline RITUXVAS & Central randomisation & Yes & No & No & NS & NS & Yes & 100 \\
\hline
\end{tabular}

Allocation concealment: A - adequate. B - Unclear. NS Not Stated. 
This investigation is currently under way as part of the PEXIVAS study run by the European Vasculitis Study Group. A cost effectiveness analysis is also required for the use of plasma exchange when compared with methylprednisolone. Clearly there is a large difference between the two therapies in terms of cost, ease of use and complications of therapy. As the population of patients evolves over time and patients with milder disease are treated, it is highly likely that any cost effectiveness analysis would suggest that a minor benefit potentially conferred by plasma exchange would come at too great a cost when compared with IV methylprednisolone. The need for dialysis after remission induction treatment with cyclophosphamide requires some clarification. Current studies suggest that pulse CPA may leave more patients on dialysis unnecessarily.

The use of MMF for remission induction is being examined in the current MYCYC study by the European Vasculitis Study group. This is a randomised study comparing oral MMF with intravenous cyclophosphamide. This will give further insight into this area with a population of patients which is likely to have a higher proportion of PR3 ANCA positive patients.

A cost effectiveness analysis is also likely to be required for rituximab. Initial results suggest that there is no major benefit in terms of efficacy with rituximab compared with cyclophosphamide. Cost savings may be evident in avoidance of adverse events but this requires further study.

\section{Appendix}

\section{Search methods for identification of studies}

Relevant studies were obtained form the following sources

1. The Cochrane Renal Group's specialised register and the Cochrane Central Register of Controlled Trials (CENTRAL) in The Cochrane Library, (Issue 1, 2008). CENTRAL and the Renal Groups specialised register contain the hand searched results of conference proceedings from general and speciality meetings. This is an ongoing activity across the Cochrane Collaboration and is both retrospective and prospective (Master List 2007, http://apps1.jhsph.edu/ cochrane/masterlist.asp). Therefore we will not specifically search conference proceedings. Please refer to The Cochrane Renal Group's Module in The Cochrane Library for the most up-to-date list of conference proceedings (Renal Group 2008).

2. MEDLINE (1966 to July 2009) using the optimally sensitive strategy developed for the Cochrane Collaboration for the identification of RCTs ([27]) together with a search strategy developed with input from the Cochrane Renal Group's Trial Search Co-ordinator. 3. EMBASE (1980 to July 2009) using a search strategy adapted from that developed for the Cochrane Col- laboration for the identification of RCTs ([28]) together with the a search strategy developed with input from the Cochrane Renal Group's Trial Search Co-ordinator.

4. Reference lists of nephrology textbooks, review articles and relevant studies.

5. Letters seeking information about unpublished or incomplete studies to investigators known to be involved in previous studies. Letters seeking information about unpublished or incomplete studies to investigators known to be involved in previous studies.

Database Search terms $\# 1$ wegener" \#2 (rapid" near progress" near glomeruloneph $\left.^{*}\right)$ or (crescent" near glomeruloneph*) \#3 glomerulonephritis \#4 (antineutrophil near cytoplasmic)

CENTRAL \#5 vasculitis \#6 goodpasture \#7 polyarteritis $\# 8$ (acute near glomeruloneph*) $\# 9$ (\#1 or \#2 or \#3 or \#4 or \#5 or \#6 or \#7 or \#8) 1. exp WEGENER'S GRANULOMATOSIS/

2. exp Antibodies, Antineutrophil Cytoplasmic/or exp Vasculitis/or exp Glomerulonephritis/or exp Polyarteritis Nodosa/

3. rapidly progressive glomerulonephritis.ti.

4. exp GOODPASTURE SYNDROME/

5. vasculitis.tw. (12821)

6. polyarteritis.tw. (1928)

7. wegener\$.tw. (3673)

8. (rapid\$ adj25 progress\$ adj25 glomeruloneph\$).ti,ab. (906)

9. or/1-8 (86982)

10. randomized controlled trial.pt. (189812)

11. controlled clinical trial.pt. (66435)

12. randomized controlled trials/(32645)

13. random allocation/(50890)

14. double blind method/(78368)

15. single blind method/(8139)

MEDLINE 16. or/10-15 (321286)

17. animals/not (animals/and human/)

(2792757)

18. 16 not 17 (304657)

19. clinical trial.pt. (383960)

20. exp clinical trials/(155138)

21.(clinic\$ adj25 trial\$).ti,ab. (99992)

22. cross-over studies/(14545)

23. (crossover or cross-over or cross over).tw. (32414)

24. ((singl\$ or doubl\$ or trebl\$ or tripl\$) adj25 (blind\$ or mask\$)).ti,ab. (77495)

25. placebos/(23040) 
26. placebo\$.ti,ab. (84360)

27. random\$.ti,ab. (285800)

28. research design/(38137)

29. or/19-28 (683077)

30.29 not 17 (633481)

31.18 or 30 (643563)

32.9 and 31 (3934)

1. exp wegener's granulomatosis/or wegener's granulomatosis.mp.

2. exp Rapidly progressive glomerulonephritis/or rapidly progressive glomerulonephritis.mp.

3. exp neutrophil cytoplasmic antibody/or neutrophil cytoplasmic antibody.mp.

4. exp vasculitis/or vasculitis.mp.

5. exp goodpasture syndrome/or goodpasture syndrome.mp.

6. exp polyarteritis nodosa/or polyarteritis nodosa.mp.

7. exp acute glomerulonephritis/or acute glomerulonephritis.mp.

8. exp proliferative glomerulonephritis/or proliferative glomerulonephritis.mp.

9. exp glomerular basement membrane antibody/or glomerular basement membrane antibody.mp.

10. exp glomerular basement membrane/or glomerular basement membrane.mp.

11. or/1-10

12. exp clinical trial/

13. evidence based medicine/

14. outcomes research/

15. crossover procedure/

EMBASE 16. double blind procedure/

17. single blind procedure/

18. prospective study/

19. exp comparative study/

20. placebo/

21. "evaluation and follow up"/

22. follow up/

23. randomization/

24. or/12-23

25. controlled study/not case control study/

26. or/24-25

27. (clinic $\$$ adj 5 trial\$).ti,ab.

28. ((singl\$ or doubl\$ or trebl\$ or tripl\$) adj

(blind $\$$ or mask\$)).ti,ab.

29. random\$.ti,ab.

30. placebo\$.ti,ab.

31. or/27-30

32. or $/ 24,31$

33. limit 32 to human

34.11 and 33

\section{Abbreviations}

AAV: ANCA associated vasculitis; AKF: acute kidney failure; ANCA: Anti-neutrophil cytoplasmic antibody; ARF: acute renal failure; AZA: Azathioprine; BVAS: Birmingham Vasculitis Activity Score; Cl: Confidence interval; CPA: cyclophosphamide; GBM: glomerular basement membrane; IVIg: Intravenous Immunoglobulin; MD: mean difference; MMF: Mycophenolate Mofetil; MPO: Myeloperoxidase; MTX: Methotrexate; NNH: number needed to harm; NNT: number needed to treat; PR3: Proteinase 3; RCT: randomised controlled trial; RPGN: rapidly progressive glomerulonephritis; RR: relative risk; RRT: renal replacement therapy; SCr: Serum Creatinine; SMD: standardised mean difference; TNF: Tumour necrosis factor; WG: Wegener's Granulomatosis.

\section{Competing interests}

The authors declare that they have no competing interests.

\section{Authors' contributions}

GW was involved in the design of the study, conducted the literature searches, obtained and analysed studies and wrote the draft and final versions of the paper. NW was involved in the design of the study conducted the literature searches, obtained and analysed studies and edited the draft and final versions of the paper. JC was involved in the design of the study, adjudication on disagreements in data analysis and editing of draft and final versions of the paper. All authors have read and approved the final manuscript.

\section{Author Details}

ISenior Staff Specialist, The Canberra Hospital, Canberra, Australia. Hon. Senior Lecturer, Australian National University Medical School, Canberra, Australia, 2Managing Editor, Cochrane Renal Group, Centre for Kidney Research, Westmead, Australia and 'The Children's Hospital at Westmead, Sydney, Australia School of Public Health, University of Sydney, Sydney, Australia

Received: 21 September 2009 Accepted: 24 June 2010

Published: 24 June 2010

References

1. Savage CO, Harper L, Adu D: Primary systemic vasculitis. Lancet 1997, 349:553-558.

2. Seo P, Stone JH: The antineutrophil cytoplasmic antibody-associated vasculitides. Am J Med 2004, 117:39-50.

3. Jennette J, Falk R: Renal and systemic vasculitis. Comprehensive clinical nephrology. Mosby 2nd edition. 2003:341-357.

4. Jennette JC, Falk RJ: New insight into the pathogenesis of vasculitis associated with antineutrophil cytoplasmic autoantibodies. Curr Opin Rheumatol 2008, 20:55-60.

5. Bolton WK, Sturgill BC: Methylprednisolone therapy for acute crescentic rapidly progressive glomerulonephritis. Am J Nephrol 1989, 9:368-375.

6. Lockwood CM, Rees AJ, Pearson TA, Evans DJ, Peters DK, Wilson CB: Immunosuppression and plasma-exchange in the treatment of Goodpasture's syndrome. Lancet 1976, 1:711-715.

7. Pusey CD, Rees AJ, Evans DJ, Peters DK, Lockwood CM: Plasma exchange in focal necrotizing glomerulonephritis without anti-GBM antibodies. Kidney Int 1991, 40:757-763.

8. Rondeau E, Levy M, Dosquet P, Ruedin P, Mougenot B, Kanfer A, Sraer JD: Plasma exchange and immunosuppression for rapidly progressive glomerulonephritis: prognosis and complications. Nephrol Dial Transplant 1989, 4:196-200.

9. Lapraik C, Watts R, Bacon P, Carruthers D, Chakravarty K, D'Cruz D, Guillevin L, Harper L, Jayne D, Luqmani R, Mooney J, Scott D: BSR and BHPR guidelines for the management of adults with ANCA associated vasculitis. Rheumatology (Oxford) 2007, 46:1615-1616.

10. Mukhtyar C, Guillevin L, Cid MC, Dasgupta B, de Groot K, Gross W, Hauser T, Hellmich B, Jayne D, Kallenberg CGM, Merkel PA, Raspe H, Salvarani C, Scott DGl, Stegeman C, Watts R, Westman K, Witter J, Yazici H, Luqmani R: EULAR recommendations for the management of primary small and medium vessel vasculitis. Ann Rheum Dis 2009, 68:310-317.

11. Menahem S, Hiremagalur B, Mudge D, Toussaint N, Walters G: The CARI guidelines. 25 Induction and maintenance therapy in ANCA-associated systemic vasculitis. Nephrology (Carlton) 2008, 13(Suppl 2):S24-36.

12. Jayne $D$ : Challenges in the management of microscopic polyangiitis: past, present and future. Curr Opin Rheumatol 2008, 20:3-9. 
13. de Groot K, Adu D, Savage CO: The value of pulse cyclophosphamide in ANCA-associated vasculitis: meta-analysis and critical review. Nephrol Dial Transplant 2001, 16:2018-2027.

14. Kerr PG, Chadban SJ, Atkins RC: Is there a role for plasma exchange in rapidly progressive glomerulonephritis? Nephrology 2001, 6:141-143.

15. Levy JB, Winearls CG: Rapidly progressive glomerulonephritis: what should be first-line therapy? Nephron 1994, 67:402-407.

16. Jayne DR, Chapel H, Adu D, Misbah S, O'Donoghue D, Scott D, Lockwood CM: Intravenous immunoglobulin for ANCA-associated systemic vasculitis with persistent disease activity. QJM 2000, 93:433-439

17. Nowack R, Birck R, van der Woude FJ: Mycophenolate mofetil for systemic vasculitis and IgA nephropathy. Lancet 1997, 349:774.

18. Tervaert JW, Stegeman CA, Kallenberg CG: Novel therapies for antineutrophil cytoplasmic antibody-associated vasculitis. Curr Opin Nephrol Hypertens 2001, 10:211-217.

19. Walters G, Willis NS, Craig JC: Interventions for renal vasculitis in adults. Cochrane Database Syst Rev 2008:CD003232.

20. de Groot K, Harper L, Jayne DRW, Flores Suarez LF, Gregorini G, Gross WL, Luqmani R, Pusey CD, Rasmussen N, Sinico RA, Tesar V, Vanhille P, Westman K, Savage COS: Pulse versus daily oral cyclophosphamide for induction of remission in antineutrophil cytoplasmic antibodyassociated vasculitis: a randomized trial. Ann Intern Med 2009, 150:670-680.

21. Hu W, Liu C, Xie H, Chen H, Liu Z, Li L: Mycophenolate mofetil versus cyclophosphamide for inducing remission of ANCA vasculitis with moderate renal 26 involvement. Nephrol Dial Transplant 2008, 23:1307-1312

22. Metzler C, Miehle N, Manger K, Iking-Konert C, de Groot K, Hellmich B, Gross WL, Reinhold-Keller E: Elevated relapse rate under oral methotrexate versus leflunomide for maintenance of remission in Wegener's granulomatosis. Rheumatology (Oxford) 2007, 46:1087-1091

23. Pagnoux C, Mahr A, Hamidou MA, Boffa J, Ruivard M, Ducroix J, Kyndt X, Lifermann F, Papo T, Lambert M, Le Noach J, Khellaf M, Merrien D, Puéchal X, Vinzio S, Cohen P, Mouthon L, Cordier J, Guillevin L: Azathioprine or methotrexate maintenance for ANCA-associated vasculitis. N Engl J Med 2008, 359:2790-2803.

24. Stone J, Merkel P, Seo P, Spiera R, Langford C, Hoffman G, Kallenberg C, William St Clair E, Fessler B, Tchao N, Ding L, Webber L, Ikle D, Weitzenkamp D, Wu W, Brunetta P, Seismundo L, Fervenza F, Keogh K, Kissin E, Mieras K, Monach P, Peikert T, Stegeman C, Ytterberg S, Specks U: Rituximab versus cyclophosphamide for induction of remission of anca associated vasculitis: a randomized controlled trial (rave) [abstract]. American College of Rheumatology Annual Meeting. Philadelphia 2009

25. Jones R, Walsh M, Jayne D, for the European Vasculitis Study Group: Randomised trial of rituximab versus cyclophophamide for anca associated renal vasculitis: rituxvas [abstract]. Annual Scientific Meeting of the American Society of Nephrology 2008

26. The Wegener's Granulomatosis Etanercept Trial (WGET) Research Group: Etanercept plus standard therapy for Wegener's granulomatosis. $\mathrm{N}$ Engl J Med 2005, 352:351-361.

27. Dickersin K, Scherer R, Lefebvre C: Identifying relevant studies for systematic reviews. BMJ 1994, 309:1286-1291.

28. Lefebvre C, McDonald S: Development of a Sensitive Search Strategy for Reports of Randomized Controlled Trials in EMBASE. Fourth International Cochrane Colloquium; Adelaide 1996.

29. Cole E, Cattran D, Magil A, Greenwood C, Churchill D, Sutton D, Clark W, Morrin P, Posen G, Bernstein K: A prospective randomized trial of plasma exchange as additive therapy in idiopathic crescentic glomerulonephritis. The Canadian Apheresis Study Group. Am J Kidney Dis 1992, 20:261-269.

30. Glöckner WM, Sieberth HG, Wichmann HE, Backes E, Bambauer R, Boesken WH, Bohle A, Daul A, Graben N, Keller F: Plasma exchange and immunosuppression in rapidly progressive glomerulonephritis: a controlled, multi-center study. Clin Nephrol 1988, 29:1-8.

31. Jayne DRW, Gaskin G, Rasmussen N, Abramowicz D, Ferrario F, Guillevin L, Mirapeix E, Savage COS, Sinico RA, Stegeman CA, Westman KW, van der Woude FJ, de Lind van Wijngaarden RAF, Pusey CD: Randomized trial of plasma exchange or high-dosage methylprednisolone as adjunctive therapy for severe renal vasculitis. J Am Soc Nephrol 2007, 18:2180-2188.
32. Mauri J, Gonzalez M, Poveda R: Therapeutic plasma exchange in the treatment of rapidly progressive glomerulonphritis. Plasma therapy and transfusion technology 1985, 6:587-591.

33. Rifle G, Chalopin JM, Zech P, Deteix P, Ducret F, Vialtel P, Cordonnier D: Treatment of idiopathic acute crescentic glomerulonephritis by immunodepression and plasma-exchanges. A prospective randomised study. Proc Eur Dial Transplant Assoc 1981, 18:493-502.

34. Adu D, Pall A, Luqmani RA, Richards NT, Howie AJ, Emery P, Michael J, Savage CO, Bacon PA: Controlled trial of pulse versus continuous prednisolone and cyclophosphamide in the treatment of systemic vasculitis. QJM 1997, 90:401-409.

35. Guillevin L, Cordier JF, Lhote F, Cohen P, Jarrousse B, Royer I, Lesavre P, Jacquot $C$, Bindi P, Bielefeld P, Desson JF, Détrée F, Dubois A, Hachulla E, Hoen B, Jacomy D, Seigneuric C, Lauque D, Stern M, Longy-Boursier M: A prospective, multicenter, randomized trial comparing steroids and pulse cyclophosphamide versus steroids and oral cyclophosphamide in the treatment of generalized Wegener's granulomatosis. Arthritis Rheum 1997, 40:2187-2198.

36. Haubitz M, Schellong S, Göbel U, Schurek HJ, Schaumann D, Koch KM, Brunkhorst R: Intravenous pulse administration of cyclophosphamide versus daily oral treatment in patients with antineutrophil cytoplasmic antibody-associated vasculitis and renal involvement: a prospective, randomized study. Arthritis Rheum 1998, 41:1835-1844.

37. Furuta T, Hotta O, Yusa N, Horigome I, Chiba S, Taguma Y: Lymphocytapheresis to treat rapidly progressive glomerulonephritis: a randomised comparison with steroid-pulse treatment. Lancet 1998, 352:203-204.

38. Stegmayr BG, Almroth G, Berlin G, Fehrman I, Kurkus J, Norda R, Olander R, Sterner G, Thysell H, Wikström B, Wirén JE: Plasma exchange or immunoadsorption in patients with rapidly progressive crescentic glomerulonephritis. A Swedish multi-center study. Int J ArtifOrgans 1999, 22:81-87.

39. Jayne D, Rasmussen N, Andrassy K, Bacon P, Tervaert JWC, Dadoniené J, Ekstrand A, Gaskin G, Gregorini G, de Groot K, Gross W, Hagen EC, Mirapeix E, Pettersson E, Siegert C, Sinico A, Tesar V, Westman K, Pusey C: A randomized trial of maintenance therapy for vasculitis associated with antineutrophil cytoplasmic autoantibodies. NEng/ J Med 2003, 349:36-44.

40. Hiemstra T, Walsh M, Schmitt W, Jayne D: Randomised controlled trial of mycophenolate mofetil versus azathioprine for maintenance therapy in anca associated vasculitis (improve) [abstract]. Annual Scientific Meeting of the American Society of Nephrology 2009.

41. Stegeman CA, Tervaert JW, de Jong PE, Kallenberg CG: Trimethoprimsulfamethoxazole (co-trimoxazole) for the prevention of relapses of Wegener's granulomatosis. Dutch Co-Trimoxazole Wegener Study Group. NEngl J Med 1996, 335:16-20.

42. Rüth E, Kemper MJ, Leumann EP, Laube GF, Neuhaus TJ: Children with steroid-sensitive nephrotic syndrome come of age: long-term outcome. J Pediatr 2005, 147:202-207.

43. Bosch X, Guilabert A, Espinosa G, Mirapeix E: Treatment of antineutrophil cytoplasmic antibody associated vasculitis: a systematic review. JAMA 2007, 298:655-669.

\section{Pre-publication history}

The pre-publication history for this paper can be accessed here: http://www.biomedcentral.com/1471-2369/11/12/prepub

doi: $10.1186 / 1471-2369-11-12$

Cite this article as: Walters et al., Interventions for renal vasculitis in adults. A systematic review BMC Nephrology 2010, 11:12 